TRANSACTIONS OF THE

AMERICAN MATHEMATICAL SOCIETY

Volume 358, Number 3, Pages 1365-1401

S $0002-9947(05) 03832-8$

Article electronically published on June 21, 2005

\title{
CONTACT REDUCTION AND GROUPOID ACTIONS
}

\author{
MARCO ZAMBON AND CHENCHANG ZHU
}

\begin{abstract}
We introduce a new method to perform reduction of contact manifolds that extends Willett's and Albert's results. To carry out our reduction procedure all we need is a complete Jacobi map $J: M \rightarrow \Gamma_{0}$ from a contact manifold to a Jacobi manifold. This naturally generates the action of the contact groupoid of $\Gamma_{0}$ on $M$, and we show that the quotients of fibers $J^{-1}(x)$ by suitable Lie subgroups $\Gamma_{x}$ are either contact or locally conformal symplectic manifolds with structures induced by the one on $M$.

We show that Willett's reduced spaces are prequantizations of our reduced spaces; hence the former are completely determined by the latter. Since a symplectic manifold is prequantizable iff the symplectic form is integral, this explains why Willett's reduction can be performed only at distinguished points. As an application we obtain Kostant's prequantizations of coadjoint orbits. Finally we present several examples where we obtain classical contact manifolds as reduced spaces.
\end{abstract}

\section{INTRODUCTION}

Marsden and Weinstein introduced symplectic reduction in 1974 [MW74]. Since then, the idea of reduction has been applied in many geometric contexts. In the realm of contact geometry, two different reduction procedures for contact Hamiltonian actions were developed by Albert Alb89] in 1989 and Willett Wil02 in 2002. However neither method is as natural as the classical Marsden-Weinstein reduction: the contact structure of Albert's reduction depends on the choice of the contact 1-form; Willett's requires additional conditions on the reduction points. In this paper we perform contact reduction via contact groupoids, following the idea of Mikami and Weinstein [MW88], who generalized the classical symplectic reduction to reduction via so-called symplectic groupoids.

Our approach not only puts both Albert's and Willett's reduction into one unified framework, but also delivers a structure on the reduced space which is independent of the choice of the contact 1-form and can be performed at all points. Moreover, to carry out our reduction, we only need a "complete Jacobi map". We will elaborate below.

We first describe the way to recover Willett's reduction from ours. Given a Hamiltonian action of a group $G$ on a contact manifold $\left(M, \theta_{M}\right)$ as in [Wil02], we can associate the action of a contact groupoid on $M$, for which we are able to perform reduction. If for simplicity we assume that $G$ is compact, then our reduced

Received by the editors May 25, 2004.

2000 Mathematics Subject Classification. Primary 53D10, 53D20, 58H05.

(C)2005 American Mathematical Society 
spaces are always symplectic manifolds, and we have

Result I: (Theorem 5.4) Willett's reduced spaces are prequantizations of our reduced (via groupoids) spaces.

Since we can realize coadjoint orbits as our reduced spaces, this allows us to construct prequantizations of coadjoint orbits, hence reproducing the results of Kostant's construction Kos70. As an example with $G=U(2)$, by our reduction, we obtain certain lens spaces as prequantizations of $S^{2}$.

Let us now outline our reduction procedure via groupoids. We first have to introduce some terminology, which will be defined rigurously in Section 2 .

Groupoids are generalizations of groups and are suitable to describe geometric situations in a global fashion.

Jacobi manifolds Lic78 arise as generalizations of Poisson manifolds and include contact manifolds. Exactly as Poisson manifolds are naturally foliated by symplectic leaves, Jacobi manifolds are foliated by two kinds of leaves: the odddimensional ones are contact manifolds, and the even-dimensional ones are so-called locally conformal symplectic (l.c.s.) manifolds.

Given a Jacobi manifold, one can associate to it a contact groupoid (i.e. a groupoid with a compatible contact structure), which one can view as the "global object" corresponding to the Jacobi structure.

In analogy to the well-known fact in symplectic geometry that the moment map allows one to reconstruct the corresponding Hamiltonian action, we have the following result:

Result II (Theorem 3.8): Any complete Jacobi map $J$ which is a surjective submersion from a contact manifold $\left(M, \theta_{M}\right)$ to a Jacobi manifold $\Gamma_{0}$ naturally induces a contact groupoid action of the contact groupoid $\Gamma$ of $\Gamma_{0}$ on $M$.

Using the notation above our main result on reduction is:

Result III (Theorem 4.1): Let the contact groupoid $\Gamma$ act on $\left(M, \theta_{M}\right)$ by contact groupoid action. Suppose that $x \in \Gamma_{0}$ is a regular value of $J$ and that $\Gamma_{x}$ acts freely and properly on $J^{-1}(x)$ (here $\Gamma_{x} \subset \Gamma$ is the isotropy group at $x$ ). Then the reduced space $M_{x}:=J^{-1}(x) / \Gamma_{x}$ has an induced

(1) contact structure, if $x$ belongs to a contact leaf,

(2) conformal l.c.s. structure, if $x$ belongs to a l.c.s. leaf.

This is the point-wise version of a result about global reduction: the quotient of a contact manifold by the action of a contact groupoid is naturally a Jacobi manifold, the leaves of which are the above reduced spaces $M_{x}$ (therefore not necessarily contact). This shows that performing any natural reduction procedure on a contact manifold, one should not expect to obtain contact manifolds in general.

Notice that combining the two results above we are able to obtain contact manifolds by reduction starting with a simple piece of data, namely a complete Jacobi map, without even mentioning groupoids.

The paper is structured as follows: in Section 2 we introduce the basic terminology. In Section 3 we prove Result II and in Section 4 we prove our point-wise reduction procedure (Result III) as well as our global reduction. 
Section 5 contains the results about Willett's and Albert's reduced spaces and prequantization, and can be read independently 11 of the previous sections. Finally, in Section 6 we give some simple concrete examples (such as cosphere-bundles) of contact manifolds obtained via groupoid reduction.

In Appendix I we show that the structures on our reduced spaces do not depend on the choice of contact form $\theta_{M}$ on $M$ but only on the corresponding contact structure, and in Appendix II we explain how the conventions we adopt relate to other conventions found in the literature. We hope this will make the literature on Jacobi manifolds and contact groupoids more easily accessible.

\section{BASIC TERMINOLOGY}

In this section we introduce Jacobi manifolds and their global counterparts, namely contact groupoids.

2.1. Jacobi manifolds. A Jacobi manifold is a smooth manifold $M$ with a bivector field $\Lambda$ and a vector field $E$ such that

$$
[\Lambda, \Lambda]=2 E \wedge \Lambda, \quad[\Lambda, E]=0,
$$

where $[\cdot, \cdot]$ is the usual Schouten-Nijenhuis brackets. A Jacobi structure on $M$ is equivalent to a "local Lie algebra" structure on $C^{\infty}(M)$ in the sense of Kirillov Kir76], with the bracket

$$
\{f, g\}=\sharp \Lambda(d f, d g)+f E(g)-g E(f) \quad \forall f, g \in C^{\infty}(M) .
$$

We call it a Jacobi bracket on $C^{\infty}(M)$. It is a Lie bracket satisfying the following equation (instead of the Leibniz rule, as Poisson brackets):

$$
\left\{f_{1} f_{2}, g\right\}=f_{1}\left\{f_{2}, g\right\}+f_{2}\left\{f_{1}, g\right\}-f_{1} f_{2}\{1, g\},
$$

i.e. it is a first order differential operator on each of its arguments. If $E=0,(M, \Lambda)$ is a Poisson manifold.

Recall that a contact manifold 2 is a $(2 n+1)$-dimensional manifold equipped with a 1-form $\theta$ such that $\theta \wedge(d \theta)^{n}$ is a volume form. If $(M, \Lambda, E)$ is a Jacobi manifold such that $\Lambda^{n} \wedge E$ is nowhere 0 , then $M$ is a contact manifold with the contact 1-form $\theta$ determined by

$$
\iota(\theta) \Lambda=0, \quad \iota(E) \theta=1
$$

where $\iota$ is the contraction between differential forms and vector fields. On the other hand, given a contact manifold $(M, \theta)$, let $E$ be the Reeb vector field of $\theta$, i.e. the unique vector field satisfying

$$
\iota(E) d \theta=0, \quad \iota(E) \theta=1 .
$$

Let $\mu$ be the map $T M \rightarrow T^{*} M, \mu(X)=-\iota(X) d \theta$. Then $\mu$ is an isomorphism between $\operatorname{ker}(\theta)$ and $\operatorname{ker}(E)$, and can be extended to their exterior algebras. Let $\Lambda=\mu^{-1}(d \theta)$. (Note that if $\iota(E) d \theta=0$, then $d \theta$ can be written as $\alpha \wedge \beta$ and $\iota(E) \alpha=\iota(E) \beta=0$.) Then $E$ and $\Lambda$ satisfy (11). So a contact manifold is always a

\footnotetext{
${ }^{1}$ More precisely: Section 5 requires only the definition of contact groupoid together with two examples (Section 2.2), the definition of contact groupoid action (Definition 3.1) and the statement of our point-wise reduction result (Theorem 4.1).

2 A related concept is the following: a contact structure on the manifold $M$ is a choice of hyperplane $\mathcal{H} \subset T M$ such that locally $\mathcal{H}=\operatorname{ker}(\theta)$ for some one-form $\theta$ satisfying $\theta \wedge(d \theta)^{n} \neq 0$. In this paper all contact structures will be co-orientable, so that $\mathcal{H}$ will be the kernel of some globally defined contact one form $\theta$.
} 
Jacobi manifold [Lic78]. Notice that in this case the map $\sharp \Lambda: T^{*} M \longrightarrow T M$ given by $\sharp \Lambda(X)=\Lambda(X, \cdot)$ and the map $\mu$ above are inverses of each other when restricted to $\operatorname{ker}(\theta)$ and $\operatorname{ker}(E)$.

A locally conformal symplectic manifold (l.c.s. manifold for short) is a $2 n$ dimensional manifold equipped with a non-degenerate two-form $\Omega$ and a closed one-form $\omega$ such that $d \Omega=\omega \wedge \Omega$. To justify the terminology notice that locally $\omega=d f$ for some function $f$, and that the local conformal change $\Omega \mapsto e^{-f} \Omega$ produces a symplectic form. If $(M, \Lambda, E)$ is a Jacobi manifold such that $\Lambda^{n}$ is nowhere 0 , then $M$ is an l.c.s. manifold: the two-form $\Omega$ is defined so that the corresponding map $T M \longrightarrow T^{*} M$ is the negative inverse of $\sharp \Lambda: T^{*} M \longrightarrow T M$, and the one-form is given by $\omega=\Omega(E, \cdot)$. Conversely, if $(\Omega, \omega)$ is an l.c.s. structure on $M$, then defining $E$ and $\Lambda$ in terms of $\Omega$ and $\omega$ as above, (11) will be satisfied.

A Jacobi manifold is always foliated by contact and locally conformal symplectic (l.c.s.) leaves dLLMP03. In fact, like a Poisson manifold, the foliation of a Jacobi manifold is also given by the distribution of the Hamiltonian vector fields

$$
X_{u}:=u E+\sharp \Lambda(d u) .
$$

The leaf through a point will be an l.c.s. (contact) leaf when $E$ lies (does not lie) in the image of $\sharp \Lambda$ at that point.

Given a nowhere vanishing smooth function $u$ on a Jacobi manifold $(M, \Lambda, E)$, a conformal change by $u$ defines a new Jacobi structure:

$$
\Lambda_{u}=u \Lambda, \quad E_{u}=u E+\sharp \Lambda(d u)=X_{u} .
$$

We call two Jacobi structures equivalent if they differ by a conformal change. A conformal Jacobi structure on a manifold is just an equivalence class of Jacobi structures 3 The relation between the Jacobi brackets induced by the $u$-twisted and the original Jacobi structures is given by

$$
\{f, g\}_{u}=u^{-1}\{u f, u g\} .
$$

The relation between the Hamiltonian vector fields is given by

$$
X_{f}^{u}=X_{u \cdot f} .
$$

A smooth map $\phi$ between Jacobi manifolds $\left(M_{1}, \Lambda_{1}, E_{1}\right)$ and $\left(M_{2}, \Lambda_{2}, E_{2}\right)$ is a Jacobi morphism if

$$
\phi_{*} \Lambda_{1}=\Lambda_{2}, \quad \phi_{*} E_{1}=E_{2},
$$

or equivalently if $\phi_{*}\left(X_{\phi^{*} f}\right)=X_{f}$ for all functions $f$ on $M_{2}$. Given $u \in C^{\infty}\left(M_{1}\right)$, a $u$ conformal Jacobi morphism from a Jacobi manifold $\left(M_{1}, \Lambda_{1}, E_{1}\right)$ to $\left(M_{2}, \Lambda_{2}, E_{2}\right)$ is a Jacobi morphism from $\left(M_{1},\left(\Lambda_{1}\right)_{u},\left(E_{1}\right)_{u}\right)$ to $\left(M_{2}, \Lambda_{2}, E_{2}\right)$.

2.2. Contact groupoids. Before introducing contact groupoids, let us fix our conventions about Lie groupoids [CdSW99], MM03]. Throughout the paper $\Gamma \underset{\mathbf{t}}{\stackrel{\mathbf{s}}{\rightrightarrows}} \Gamma_{0}$ will be a Lie (contact) groupoid, its Lie algebroid will be identified with $\operatorname{ker}(d \mathbf{t})$, and the multiplication $\omega$ will be defined on the fiber-product $\Gamma_{\mathbf{s}} \times_{\mathbf{t}} \Gamma:=\{(g, h) \mid \mathbf{s}(g)=$ $\mathbf{t}(h), g, h \in \Gamma\}$ 出

\footnotetext{
${ }^{3}$ Clearly a conformal contact manifold is just a manifold with a coorientable contact structure.

${ }^{4}$ Also see Definition 3.1
} 
Definition 2.1. A contact groupoid KSB93 is a Lie groupoid $\Gamma \rightrightarrows \Gamma_{0}$ equipped with a contact 1-form $\theta$ and a smooth non-vanishing function $f$, such that on $\Gamma_{\mathbf{s}} \times_{\mathbf{t}} \Gamma$ we have

$$
\omega^{*} \theta=p r_{2}^{*} f \cdot p r_{1}^{*} \theta+p r_{2}^{*} \theta
$$

where $p r_{j}$ is the projection from $\Gamma_{\mathbf{s}} \times_{\mathbf{t}} \Gamma \subset \Gamma \times \Gamma$ onto the $j$-th factor.

Remark 2.2. Let us recall some useful facts from [KSB93, Daz97, and [CZ] about contact groupoids:

a) A contact groupoid $\Gamma \rightrightarrows \Gamma_{0}$ induces a Jacobi structure on its base manifold. We denote the vector fields and bivector fields defining the Jacobi structures by $E_{\Gamma}, E_{0}$ and $\Lambda_{\Gamma}, \Lambda_{0}$, respectively.

b) With respect to this Jacobi structure the source map $\mathbf{s}$ is Jacobi morphism and the target $\mathbf{t}$ is $-f$-conformal Jacobi (see also Appendix II).

c) On the other hand, for certain Jacobi manifolds $\Gamma_{0}$, there is a unique contact groupoid $\Gamma \rightrightarrows \Gamma_{0}$ with connected, simply connected t-fibers (or equivalently, s-fibres) satisfying b). In this case, we call $\Gamma_{0}$ integrable. Integrability conditions of Jacobi manifolds are studied in detail in [CZ].

d) Furthermore, at any $g \in \Gamma$, the kernels of $T \mathbf{s}$ and $T \mathbf{t}$ are given by (Daz97])

$$
\begin{array}{r}
\operatorname{ker} T_{g} \mathbf{t}=\left\{X_{\mathbf{s}^{*} u}(g): u \in C^{\infty}\left(\Gamma_{0}\right)\right\}, \\
\operatorname{ker} T_{g} \mathbf{s}=\left\{X_{f \cdot \mathbf{t}^{*} u}(g): u \in C^{\infty}\left(\Gamma_{0}\right)\right\} .
\end{array}
$$

e) The function $f$ in Definition2.1 is automatically multiplicative, i.e. $f(g h)=$ $f(g) f(h)$ for all composable $g, h \in \Gamma$. Furthemore, $f$ satisfies $d f\left(E_{\Gamma}\right)=0$.

f) The constructions of this paper admit a version that involves only contact structures and is independent of contact forms. Interested readers are referred to Appendix I.

Example 2.3 (Contact groupoid of $S\left(\mathfrak{g}^{*}\right)$ ). For a Lie group $G$, let $\mathfrak{g}^{*}$ be the dual of its Lie algebra $\mathfrak{g}$. Choose any Riemannian metric on it; then the quotient space $S\left(\mathfrak{g}^{*}\right):=\left(\mathfrak{g}^{*}-0\right) / \mathbb{R}^{+}$is a Jacobi manifold $[$([Lic78] and dLLMP03]). The "Poissonization" of $S\left(\mathfrak{g}^{*}\right)$ is the Poisson manifold $\mathfrak{g}^{*}-0$.

In particular, when $G$ is compact, we can choose a bi-invariant metric, then $S\left(\mathfrak{g}^{*}\right)$ can be embedded in $\mathfrak{g}^{*}$ as the unit sphere which is Poisson with the restricted Poisson structure because all the symplectic leaves - the coadjoint orbits - will stay in the sphere. In this case, the contact groupoid of $S\left(\mathfrak{g}^{*}\right)$ is $\left(U^{*} G, \theta_{c}, 1\right)$, where $U^{*} G$ is the set of covectors of length one and $\theta_{c}$ is the restriction of the canonical 1-form to the cosphere bundle (see Example 6.8 of [BCWZar]). Recall that the groupoid structure is given by

$$
\begin{array}{r}
\mathbf{t}(\bar{\eta})=R_{g}^{*} \bar{\eta}, \quad \mathbf{s}(\bar{\eta})=L_{g}^{*} \bar{\eta}, \\
\bar{\eta}_{1} \cdot \bar{\eta}_{2}=\frac{1}{2}\left(R_{g_{2}^{-1}}^{*} \bar{\eta}_{1}+L_{g_{1}^{-1}}^{*} \bar{\eta}_{2}\right) \in U_{g_{1} g_{2}}^{*} G,
\end{array}
$$

where $\bar{\eta} \in U_{g}^{*} G, \bar{\eta}_{i} \in U_{g_{i}}^{*} G$, and $R_{g}, L_{g}$ denote the right and left translations by $g$. Identifying $U^{*} G$ and $S\left(\mathfrak{g}^{*}\right) \rtimes G$ by right translations, i.e. identifying a covector $R_{g^{-1}}^{*} \xi$ at $g$ with $(\xi, g)$, the contact groupoid structure is given by

$$
\begin{aligned}
\mathbf{t}(\xi, g) & =\xi, & \mathbf{s}(\xi, g) & =L_{g}^{*} R_{g^{-1}}^{*} \xi \\
\left(\xi_{1}, g_{1}\right) \cdot\left(\xi_{2}, g_{2}\right) & =\left(\xi_{1}, g_{1} g_{2}\right), & \theta_{c}(\delta \xi, \delta g)_{(\xi, g)} & =\left\langle\xi, R_{g^{-1}} \delta g\right\rangle .
\end{aligned}
$$

\footnotetext{
${ }^{5}$ Its structure depends on the metric.
} 
For a general Lie group $G$, the symplectification of the quotient cosphere bundle $S^{*} G:=\left(T^{*} G-G\right) / \mathbb{R}^{+}$is $T^{*} G-G$, which is exactly the symplectic groupoid of $\mathfrak{g}^{*}-0$ - the Poissonization of $S\left(\mathfrak{g}^{*}\right)$. By the main result in [CZ $\left(T^{*} G-G\right) / \mathbb{R}^{+}$is the contact groupoid of $S\left(\mathfrak{g}^{*}\right)$ with contact 1-form $\theta$ and function $f$ which, using the trivilization by right translations, are given by

$$
\theta(\delta \xi, \delta g)_{([\xi], g)}=\frac{\left\langle\xi, R_{g^{-1} *} \delta g\right\rangle}{\left\|L_{g}^{*} R_{g^{-1}}^{*} \xi\right\|}, \quad f([\xi], g)=\frac{\|\xi\|}{\left\|L_{g}^{*} R_{g^{-1}}^{*} \xi\right\|},
$$

where [.] denotes the equivalence class under the $\mathbb{R}^{+}$action. The groupoid structure is inherited from $T^{*} G$ (very similar to the compact case we have just presented and also see the examples in [CZ]).

Example 2.4 (Contact groupoid of $\mathfrak{g}^{*}$ ). Using the same notation as the last example, we view the Poisson manifold $\mathfrak{g}^{*}$ as a Jacobi manifold. Then the contact groupoid of $\mathfrak{g}^{*}$ is $\left(T^{*} G \times \mathbb{R}, 1, \theta_{c}+d r\right)$, where $\theta_{c}$ is the canonical 1-form on $T^{*} G$ and $d r$ is the 1 -form on $\mathbb{R}$. (The proof is similar to the one of Theorem $4.8 \mathrm{in}[\mathrm{CZ}]$ ).

Identifying $T^{*} G \times \mathbb{R}$ with $\mathfrak{g}^{*} \rtimes G \times \mathbb{R}$ by right translation, the groupoid structure is given by

$$
\begin{array}{r}
\mathbf{t}(\xi, g, r)=\xi, \quad \mathbf{s}(\xi, g, r)=L_{g}^{*} R_{g^{-1}}^{*} \xi, \\
\left(\xi_{1}, g_{1}, r_{1}\right) \cdot\left(\xi_{2}, g_{2}, r_{2}\right)=\left(\xi_{1}, g_{1} g_{2}, r_{1}+r_{2}\right) .
\end{array}
$$

\section{Contact groupoid aCtions And COntact Realizations}

In this section, we introduce contact groupoid action and show that they can be encoded by their "moment maps". To this aim we present a new concept-contact realizations. At the end of this section we introduce the $f$-multiplicative functions, which are also called reduction functions, to allow us to perform reductions in the next section.

3.1. Contact groupoid actions and moment maps. Just as groups, groupoids can also act on a manifold, though in a more subtle way:

Definition 3.1 ((Contact) Groupoid Action). Let $\Gamma \rightrightarrows \Gamma_{0}$ be a Lie groupoid, and let $M$ be a manifold equipped with a moment map $J: M \rightarrow \Gamma_{0}$. A groupoid (right) action of $\Gamma$ on $M$ is a map

$$
\Phi: M_{J} \times_{t} \Gamma \rightarrow M, \quad(m, g) \mapsto \Phi(m, g):=m \cdot g
$$

such that

i) $J(m \cdot g)=\mathbf{s}(g)$,

ii) $(m \cdot g) \cdot h=m \cdot g h$,

iii) $m \cdot J(m)=m$, with the identification $\Gamma_{0} \hookrightarrow \Gamma$ as the unit elements.

Here $M_{J} \times_{\mathbf{t}} \Gamma$ is the fibre product over $\Gamma_{0}$, that is, the pre-image of the diagonal under the map $(J, \mathbf{t}): M \times \Gamma \rightarrow \Gamma_{0} \times \Gamma_{0}$. Since $\mathbf{t}$ is a submersion (because $\Gamma$ is a Lie groupoid), $M_{J} \times_{\mathbf{t}} \Gamma$ is a smooth manifold.

Given a contact groupoid $\left(\Gamma, \theta_{\Gamma}, f\right)$ and a contact manifold $\left(M, \theta_{M}\right), \Phi$ is a contact groupoid (right) action if it is a groupoid action and additionally satisfies

$$
\Phi^{*}\left(\theta_{M}\right)=p r_{\Gamma}^{*}(f) p r_{M}^{*}\left(\theta_{M}\right)+p r_{\Gamma}^{*}\left(\theta_{\Gamma}\right),
$$

where $p r_{\Gamma}$ and $p r_{M}$ are projections from $M_{J} \times_{\mathbf{t}} \Gamma$ to $\Gamma$ and $M$, respectively. This definition is modelled so that the action of a contact groupoid on itself by right multiplication is a contact groupoid action (see equation (3)). 
Remark 3.2. i) The moment map $J: \Gamma \rightarrow M$ of any groupoid action is equivariant (MW88]).

ii) A groupoid action is free if there is no fixed point; a groupoid action is proper if the following map is proper:

$$
M_{J} \times_{\mathbf{t}} \Gamma \rightarrow M \times M \text { given by }(m, g) \mapsto(m, m \cdot g) \text {. }
$$

The following lemma gives an alternative, more geometrical characterization of contact groupoid action.

Lemma 3.3. Let $\Phi$ be an action of the contact groupoid $\left(\Gamma, \theta_{\Gamma}, f\right)$ on the contact manifold $\left(M, \theta_{M}\right)$. Then $\Phi$ is a contact groupoid action if and only if the graph of $\Phi$ is a Legendrian submanifold of the contact manifold

$$
\left(M \times \mathbb{R} \times \Gamma \times \mathbb{R} \times M,-f e^{-a} \theta_{1}-e^{-b} \theta_{\Gamma}+\theta_{3}\right),
$$

where $a$ and $b$ denote the coordinates on the first and second copy of $\mathbb{R}$, respectively, $\theta_{1}$ and $\theta_{3}$ are the contact forms on the first and last copy of $M$ respectively.

Proof. We denote the one form on $M \times \mathbb{R} \times \Gamma \times \mathbb{R} \times M$ by $\Theta$. Then

$$
\begin{aligned}
d \Theta= & -e^{-a} d f \wedge \theta_{1}+f e^{-a} d a \wedge \theta_{1}-f e^{-a} d \theta_{1} \\
& +e^{-b} d b \wedge \theta_{\Gamma}-e^{-b} d \theta_{\Gamma}+d \theta_{3} .
\end{aligned}
$$

One can easily check that the Reeb vector field $E_{3}$ of the last copy of $M$ lies in the kernel of $d \Theta$, and that on the tangent space at any point of $M \times \mathbb{R} \times \Gamma \times \mathbb{R} \times M$, the form $d \Theta$ is non-degenerate on a complement of $\operatorname{span}\left\{E_{3}\right\}$. Therefore $\Theta$ is indeed a contact form (with Reeb vector field $E_{3}$ ).

Denote the graph of $\Phi$ by $\mathcal{A}$. Then the natural embedding of $\mathcal{A}$ into $M \times \mathbb{R} \times \Gamma \times$ $\mathbb{R} \times M$ is given by $(m, g, \Phi(m, g)) \mapsto(m, 0, g, 0, \Phi(m, g))$. Suppose $\Gamma$ has dimension $2 n+1$ and $M$ dimension $k$. Since $\mathbf{t}: \Gamma \rightarrow \Gamma_{0}$ is a submersion, by a simple dimension counting, $\mathcal{A}$ has the same dimension as $\Gamma_{J} \times_{\mathbf{t}} M$, which has dimension $n+k+1$. Since $M \times \mathbb{R} \times \Gamma \times \mathbb{R} \times M$ has dimension $2 n+2 k+3$, the embedding of $\mathcal{A}$ is Legendrian if and only if $\mathcal{A}$ is tangent to the contact distribution $\operatorname{ker} \Theta$. It is not hard to see that this condition is equivalent to $\Phi$ being a contact groupoid action from the equation

$$
\Theta\left(Y, 0, V, 0, \Phi_{*}(Y, V)\right)=-f(g) \theta_{M}(Y)-\theta_{\Gamma}(V)+\theta_{M}\left(\Phi_{*}(Y, V)\right),
$$

where $Y \in T_{m} M$ and $V \in T_{g} \Gamma$ for which $\Phi_{*}(Y, V)$ is defined.

The moment map of a contact groupoid action has the following nice property:

Proposition 3.4. The moment map $J: M \rightarrow \Gamma_{0}$ of any contact groupoid action is a Jacobi map.

Proof. We claim that it is enough to show that $\left(0, X_{\mathbf{s}^{*} u}, X_{J^{*} u}\right)$ is in $T \mathcal{A}$, where $\mathcal{A}$ denotes the graph of $\Phi$ and we identify it as its natural embedding as in Lemma 3.3. This is equivalent to

$$
0(m) \cdot X_{\mathbf{s}^{*} u}(g)=X_{J^{*} u}(m \cdot g)
$$

for all $(m, g) \in M_{J} \times_{\mathbf{t}} \Gamma$, and $u \in C^{\infty}\left(\Gamma_{0}\right)$, where $0(m)$ denotes the zero vector in $T_{m} M$. By the definition of groupoid action and since $\mathbf{s}$ is a Jacobi map, it follows that

$$
J_{*}\left(X_{J^{*} u}(m \cdot g)\right)=\mathbf{s}_{*}\left(X_{\mathbf{s}^{*} u}(g)\right)=X_{u}(\mathbf{s}(g))=X_{u}(J(m \cdot g)) .
$$


Therefore we have $J_{*}\left(X_{J^{*} u}\right)=X_{u}$ for all $u \in C^{\infty}\left(\Gamma_{0}\right)$, which is equivalent to $J$ being a Jacobi map.

Let $(Y, V) \in T_{(m, g)}\left(M_{\mathbf{t}} \times_{J} \Gamma\right)$. Using the 2-form $d \Theta$ from Lemma 3.3, we have at point $(m, 0, g, 0, m \cdot g)$,

$$
\begin{aligned}
& d \Theta\left(\left(0(m), 0, X_{\mathbf{s}^{*} u}(g), 0, X_{J^{*} u}(m \cdot g)\right),(Y, 0, V, 0, Y \cdot V)\right) \\
= & -X_{\mathbf{s}^{*} u}(f) \theta_{M}(Y)-d \theta_{\Gamma}\left(X_{\mathbf{s}^{*} u}, V\right)+d \theta_{M}\left(X_{J^{*} u}, Y \cdot V\right) \\
= & \left(f(g) \theta_{M}(Y)+\theta_{\Gamma}(V)\right) \cdot d u\left(\left(J_{*} E_{M}\right)-E_{0}\right) .
\end{aligned}
$$

In the last equation, we use the fact from Daz97 that $\left\{X_{f}, X_{\mathbf{s}^{*} u}\right\}=0$, and the fact that $J_{*}(Y \cdot V)=\mathbf{s}_{*} V$, and finally the fact that for a Hamiltonian vector field $X_{h}$ and a vector field $W, d \theta_{\Gamma}\left(X_{h}, W\right)=-d h\left(W_{\mathcal{H}}\right)$, where $W_{\mathcal{H}}=W-\theta_{\Gamma}(W) E_{\Gamma}$ is the projection of $W$ onto $\mathcal{H}=\operatorname{ker}\left(\theta_{\Gamma}\right)$. It is easy to see that $\left(0,0, X_{\mathbf{s}^{*} u}, 0, X_{J^{*} u}\right) \in \operatorname{ker} \Theta$, because

$$
-\mathbf{s}^{*} u(g)+J^{*} u(m \cdot g)=0 .
$$

$\mathcal{A}$ is embedded as a Legendrian submanifold by Lemma 3.3 and the vector field $\left(0,0, X_{\mathbf{s}^{*} u}, 0, X_{J^{*} u}\right)$ along $\mathcal{A}$ lies in $\operatorname{ker}(\Theta)$, so if it lies in $(T \mathcal{A})^{d \Theta}$ —as we will show below - then it automatically lies in $T \mathcal{A}$.

Now, if $u=1$, then (77) is clearly zero. Notice that $X_{\mathbf{s}^{*} 1}=E_{\Gamma}$ and $X_{J^{*} 1}=E_{M}$. So $\left(0,0, E_{\Gamma}, 0, E_{M}\right)$ lies in $(T \mathcal{A})^{d \Theta}$, and hence in $T \mathcal{A}$. Therefore

$$
J_{*}\left(E_{M}\right)=\mathbf{s}_{*}\left(E_{\Gamma}\right)=E_{0}
$$

which implies that (7) is 0 for all $u \in C^{\infty}\left(\Gamma_{0}\right)$. Repeating verbatim the above reasoning we conclude that $\left(0,0, X_{\mathbf{s}^{*} u}, 0, X_{J^{*} u}\right) \in T \mathcal{A}$, as claimed.

With the same set-up as the last two statements, we have the following lemma.

Lemma 3.5. The contact groupoid action is locally free at $m \in M$ iff $J$ is a submersion at $m$ and $T_{m} J^{-1}(J(m)) \not \subset \operatorname{ker}\left(\theta_{M}\right)_{m}$.

Remark 3.6. This differs from the corresponding statement for symplectic groupoid actions. In that case $J$ is a submersion iff the action is locally free. Example 6.2 and Remark 6.3 show that the two conditions above in the contact case are both neccessary.

Proof. $J$ being a submersion at $m$ is equivalent to the fact that the set $\left\{J^{*} d u_{i}(m)\right\}$ is linearly independent, where $u_{1}, \cdots, u_{n}$ are coordinate functions on $\Gamma_{0}$ vanishing at $x=J(m)$. By equation (6) the $\Gamma$-action is locally free if and only if $\operatorname{span}\left\{X_{J^{*} 1}=\right.$ $\left.E_{M}, X_{J^{*} u_{1}}, \cdots, X_{J^{*} u_{n}}\right\}$ at $m$ has dimension equal to the one of the $\mathbf{t}$-fibers, which is $n+1$.

If we assume that $J$ is a submersion, then the $J^{*} d u_{i}(m)$ 's are linearly independent. If we assume that $T J^{-1}(x) \not \subset \operatorname{ker}\left(\theta_{M}\right)_{m}$, then no non-trivial linear combination $\sum a_{i} \cdot J^{*} d u_{i}(m)$ lies in $\operatorname{ker}\left(\sharp \Lambda_{M}\right)_{m}=\operatorname{span}\left(\theta_{M}\right)_{m}$ (because $T J^{-1}(x)$ is contained in the kernel of $\sum a_{i} \cdot J^{*} d u_{i}(m)$ but not in the kernel of $\left.\theta_{M}\right)$. But this means that $\left\{X_{J^{*} u_{1}}, \cdots, X_{J^{*} u_{n}}\right\}$ is linearly independent at $m$. The independence is preserved after we add $X_{J^{*} 1}=E_{M}$ to this set, so the action is free there.

Conversely, let us assume that the action is locally free at $m$, that is, that $\left\{E_{M}, X_{J^{*} u_{1}}, \cdots, X_{J^{*} u_{n}}\right\}$ is a linearly independent set at $m$. Since $\sharp \Lambda_{M} J^{*} d u_{i}=$ $X_{J^{*} u_{i}}$, this implies that the $\left\{J^{*} u_{i}(m)\right\}$ 's are linearly independent, i.e. that $J$ is a submersion at $m$. This also implies that no non-trivial linear combination of 
the $J^{*} d u_{i}(m)$ lies in $\operatorname{ker}\left(\sharp \Lambda_{M}\right)_{m}=\operatorname{span}\left(\theta_{M}\right)_{m}$. Since $J$ is a submersion, we have $\left\{J^{*} d u_{i}\right\}=\left(T_{m} J^{-1}(x)\right)^{0}$, so this is possible only if $T_{m} J^{-1}(x) \not \subset \operatorname{ker}\left(\theta_{M}\right)_{m}$.

3.2. Contact realizations and moment maps. When exactly can a map from a contact manifold $M$ to a Jacobi manifold $\Gamma_{0}$ be realized as a moment map of some contact groupoid action? From Proposition 3.4, we know that the map must necessarily be a Jacobi map. To determine the remaining necessary conditions we introduce complete contact realizations.

Definition 3.7. A contact realization of a Jacobi manifold $\Gamma_{0}$ consists of a contact manifold $M$ together with a surjective Jacobi submersion $J: M \rightarrow \Gamma_{0}$. A contact realization is called complete if $X_{J^{*} u}$ is a complete vector field on $M$ whenever $u$ is a compactly supported function on $\Gamma_{0}$.

The remainder of this subsection is devoted to the proof of the following theorem:

Theorem 3.8. Let $M$ be a contact manifold, let $\Gamma_{0}$ be an integrable Jacobi manifold, and let $J: M \rightarrow \Gamma_{0}$ be a complete contact realization. Then $J$ induces a (right) contact groupoid action of $\Gamma$ on $M$, where $\Gamma$ is the unique contact groupoid integrating $\Gamma_{0}$ with connected, simply connected $\mathbf{t}$-fibers.

Remark 3.9. One can remove the above integrability condition on the Jacobi manifold. In fact, the existence of a complete contact realization for a Jacobi manifold is equivalent to its integrability. This will be explored in a future work.

Proof. In the first part of the proof 6 we will construct a suitable subset $L$ of $M \times \Gamma \times M$ and show that (a natural embedding of) it is Legendrian. In the second part, we will show that $L$ is the graph of a contact groupoid action.

Let $K=M \times \Gamma_{\mathbf{s}} \times{ }_{J} M$, which is $n+2 k+1$-dimensional 7 Consider the $(n+1)$ dimensional distribution

$$
D:=\left\{\left(0, X_{\mathbf{s}^{*} u}, X_{J^{*} u}\right) \mid u \in C^{\infty}\left(\Gamma_{0}\right)\right\} .
$$

Since both $\mathbf{s}$ and $J$ are Jacobi maps, $\left.(\mathbf{s}, J)_{*}\left(0, X_{\mathbf{s}^{*} u}, X_{J^{*} u}\right)\right|_{K}$ is tangent to the diagonal in $\Gamma_{0} \times \Gamma_{0}$. So $\left.D\right|_{K}$ is tangent to $K$.

Claim 1: $\left.D\right|_{K}$ defines an integrable distribution on $K$. We denote by $F$ the $(n+1)$-dimensional foliation of $K$ integrating it.

Proof: Denote by $\hat{K}$ the natural inclusion of $K$ into the $(2 k+2 n+3)$-dimensional manifold $M \times \mathbb{R} \times \Gamma \times \mathbb{R} \times M$, and let $\hat{J}=\left\{\left(m, a, g, 0, m^{\prime}\right) \mid m \in M, a \in \mathbb{R}, \mathbf{s}(g)=\right.$ $\left.J\left(m^{\prime}\right)\right\}$ (so $\operatorname{dim} \hat{J}=n+2 k+2$ and $\left.\hat{K} \subset \hat{J}\right)$. Denote by $\hat{D}$ the distribution $\{(0,0$, $\left.\left.X_{\mathbf{s}^{*} u}, 0, X_{J^{*} u}\right)\right\}$ on $M \times \mathbb{R} \times \Gamma \times \mathbb{R} \times M$. Now we adopt the notation of Lemma 3.3 and claim that

$$
\left.\hat{D}\right|_{\hat{J}}=(T \hat{J} \cap \operatorname{ker} \Theta)^{d \Theta} \cap \operatorname{ker} \Theta .
$$

Both are distributions of dimension $n+1$, so we just need to show the inclusion " $\subset$ ". A computation shows that for any tangent vector $Y$ we have

$$
\begin{aligned}
d \Theta\left(\left(0,0, X_{\mathbf{s}^{*} u}, 0, X_{J^{*} u}\right), Y\right) & =d u\left(E_{0}\right) \cdot \Theta(Y)-e^{-b} \cdot d b\left(Y_{b}\right) \cdot \mathbf{s}^{*} u \\
& -J^{*} d u\left(Y_{3}\right)+e^{-b} \mathbf{s}^{*} d u\left(Y_{\Gamma}\right),
\end{aligned}
$$

\footnotetext{
${ }^{6}$ We adapt the proofs of the analogous statements for symplectic realizations from [CDW87] and Xu91.

${ }^{7}$ Here as usual $\operatorname{dim} M=k$ and $\operatorname{dim} \Gamma=2 n+1$.
} 
where the subscripts denote the components of $Y$ analogously to the notation of Lemma 3.3. Clearly this vanishes if $Y \in T \hat{J} \cap \operatorname{ker} \Theta$. Together with the fact that $\left.\hat{D}\right|_{\hat{J}}$ is annihilated by $\Theta$, this proves equation (8). To complete the proof, we need to recall the following fact:

Fact: If $(C, \theta)$ is a contact manifold and $S$ a submanifold which satisfies the "coisotropicity" condition

$$
(T S \cap \operatorname{ker} \theta)^{d \theta} \cap \operatorname{ker} \theta \subset T S \cap \operatorname{ker} \theta,
$$

then the subbundle $(T S \cap \operatorname{ker} \theta)^{d \theta} \cap \operatorname{ker} \theta$ is integrable.

Proof: The proof is a straightforward computation using $d^{2} \theta=0$ to show that $[X, Y] \in(T S \cap \operatorname{ker} \theta)^{d \theta \cap \operatorname{ker} \theta}$ whenever $X, Y \in(T S \cap \operatorname{ker} \theta)^{d \theta} \cap \operatorname{ker} \theta \subset T S \cap \operatorname{ker} \theta$.

Since $\mathbf{s}$ and $J$ are both Jacobi maps, $\left.\hat{D}\right|_{\hat{J}} \subset T \hat{J} \cap \operatorname{ker} \Theta$. Therefore our distribution $\left.\hat{D}\right|_{\hat{J}}$ is integrable. Since $\left.\hat{D}\right|_{\hat{K}}$ is tangent to $\hat{K}$, it is also integrable, and the integrability of $\left.\hat{D}\right|_{\hat{K}}$ is clearly equivalent to the integrability of $D_{K}$.

Now define $I:=\{(m, J(m), m) \mid m \in M\}$, a $k$-dimensional submanifold of $K$. Notice that $I$ is transversal to the foliation $F$. We define

$$
L:=\prod_{x \in I} F_{x}
$$

where $F_{x}$ is the leaf of $F$ through $x$. As in Appendix 3 of [CDW87] one shows that $L$ is an immersed $(n+k+1)$-dimensional submanifold of $K$.

Claim 2: $\hat{L}$ is an immersed Legendrian submanifold of $M \times \mathbb{R} \times \Gamma \times \mathbb{R} \times M$, endowed with the contact form $\Theta$ as in Lemma 3.3.

Proof: Denote by $\hat{I}$ and $\hat{L}$ respectively the natural inclusions of $I, L \subset M \times \Gamma \times M$ into $M \times \mathbb{R} \times \Gamma \times \mathbb{R} \times M$. By contracting with $\Theta$ and $d \Theta$, one can show that the vector fields $\left(0,0, X_{\mathbf{s}^{*} u}, 0, X_{J^{*} u}\right)$ and the Hamiltonian vector field $\hat{X}_{J^{*} u-e^{-b} \mathbf{s}^{*} u}$ on $M \times \mathbb{R} \times \Gamma \times \mathbb{R} \times M$ coincide. Therefore the tangent spaces to the foliation $\hat{F}$ of $\hat{K}$ are actually spanned by Hamiltonian vector fields.

It is clear that at all points $\hat{x}$ in $\hat{I}$ the tangent space $T_{\hat{x}} \hat{L}$ is annihilated by $\Theta$ : for vectors tangent to $\hat{I}$ we have $\left(-\theta_{1}-\theta_{\Gamma}+\theta_{3}\right)\left(\delta m, 0, J_{*}(\delta m), 0, \delta m\right)=0$ because $\Gamma_{0} \subset \Gamma$ is Legendrian for $\theta_{\Gamma}$, for vectors tangent to the foliation $\hat{F}$ we clearly have $\left(-\theta_{1}-\theta_{\Gamma}+\theta_{3}\right)\left(0,0, X_{\mathbf{s}^{*} u}, 0, X_{J^{*} u}\right)=0$. A general point $\hat{y}$ of $\hat{L}$ can be joint to some $\hat{x} \in \hat{I}$ by finitely many segments of flows of vector fields of the form $\left(0,0, X_{\mathbf{s}^{*} u}, 0, X_{J^{*} u}\right)$. Since we just showed that these are Hamiltonian vector fields, their flows will preserve ker $\Theta$. Furthermore, since these vector fields are tangent to $\hat{L}$, they will preserve tangent spaces to $\hat{L}$, so we can conclude that since $T \hat{L} \subset \operatorname{ker} \Theta$ at $\hat{x}$ the same must be true at $\hat{y}$. The argument is finished by a simple dimension counting.

Claim 3: $L$ is the graph of a contact groupoid action.

Proof: Recall that $L$ was defined in such a way that any $\left(m, g, m^{\prime}\right) \in L$ can be reached from $(m, J(m), m)$ following the flows of vector fields of the form $\left(0, X_{\mathbf{s}^{*} u}\right.$, $\left.X_{J^{*} u}\right)$. Since $X_{\mathbf{S}^{*} u}$ is tangent to the t-fibers we have $J(m)=\mathbf{t}(g)$; in the next claim we will show that $L$ is the graph of a map $M_{J} \times_{\mathbf{t}} \Gamma \rightarrow M$. Now we check that conditions i)-iii) and equation (4) in Definition 3.1 are satisfied.

Since both $\mathbf{s}$ and $J$ are Jacobi maps, from the above remark about $L$ we have $\mathbf{s}(g)=J\left(m^{\prime}\right)$, i.e. i). Condition iii) is trivially satisfied, and equation (44) is satisfied because $\hat{L}$ is Legendrian in $M \times \mathbb{R} \times \Gamma \times \mathbb{R} \times M$ using Lemma 3.3 . 
To establish ii) we have to show that if $\left(m, g, m^{\prime}\right)$ and $\left(m^{\prime}, g^{\prime}, m^{\prime \prime}\right)$ lie in $L$, then $\left(m, g g^{\prime}, m^{\prime \prime}\right)$ also lies in $L$. We have $g=\phi_{t_{0}}^{\mathbf{s}^{*}} u_{0}(J(m))$, where by the symbol $\phi_{t_{0}}^{\mathbf{s}^{*}} u_{0}$ we denote a suitable flow of a collection $u_{0} \in C^{\infty}\left(\Gamma_{0}\right)$ at time $t_{0}$, and similarly for $\phi_{t_{0}}^{J^{*} u_{0}}(m)$ and $g^{\prime}=\phi_{t_{1}}^{\mathbf{s}^{*}} u_{1}\left(J\left(m^{\prime}\right)\right)$. Therefore we must have $m^{\prime \prime}=\phi_{t_{1}}^{J^{*}} u_{1} \circ \phi_{t_{0}}^{J^{*} u_{0}}(m)$. But $g g^{\prime}=g \phi_{t_{1}}^{\mathbf{s}^{*} u_{1}}(\mathbf{s}(g))=\phi_{t_{1}}^{\mathbf{s}^{*} u_{1}}(g)$ since vector fields of the form $X_{\mathbf{s}^{*} u}$ are left invariant (see Proposition 4.3 in [Daz97]), therefore $\left(m, g g^{\prime}, m^{\prime \prime}\right) \in L$.

To end the proof we still need

Claim 4: $L$ is the graph of a map $M_{J} \times_{\mathbf{t}} \Gamma \rightarrow M$.

Proof: Restrict to $L$ the obvious projections $p r_{1}$ (onto the first copy of $M$ ) and $p r_{\Gamma}$, originally defined on $M \times \Gamma \times M$, and denote them by the same symbols. We need to show that $\left(p r_{1}, p r_{\Gamma}\right)$ is a diffeomorphism of $L$ onto $M_{J} \times_{\mathbf{t}} \Gamma$, or equivalently that, for any $m \in M$, the map

$$
p r_{\Gamma}: p r_{1}^{-1}(m) \rightarrow \mathbf{t}^{-1}(J(m))
$$

is a diffeomorphism. Since $p r_{1}: L \rightarrow M$ is a submersion and $\operatorname{dim} L=n+k+1$, one sees that the domain of $p r_{\Gamma}$ has dimension $n+1$, which is the dimension of the target space.

We claim that $p r_{\Gamma}$ is surjective. Let $g \in \mathbf{t}^{-1}(J(m))$. Since $\mathbf{t}^{-1}(J(m))$ is connected and its tangent spaces are spanned by vector fields of the form $X_{\mathbf{s}^{*} u}$, we can find functions (collectively denoted $u_{0}$ ) such that a composition $\phi^{\mathbf{s}^{*} u_{0}}$ of their Hamiltonian flows maps $g$ to $\mathbf{t}(g)$, i.e. for some $t_{0}$ we have $\phi_{t_{0}}^{\mathbf{s}^{*} u_{0}}(\mathbf{t}(g))=g$. Let us denote by $\phi^{u_{0}}$ and $\phi^{J^{*} u_{0}}$ the analogously defined Hamiltonian flows on $\Gamma_{0}$ and $M$. The image of the curve $\left[0, t_{0}\right] \rightarrow \Gamma_{0}, t \mapsto \phi_{t}^{u_{0}}(\mathbf{t}(g))$ lies in a compact subset of $\Gamma_{0}$, so we may assume that all the functions that we collectively denote by $u_{0}$ have compact support. By the completeness assumption on $J$ we conclude that $\phi_{t}^{J^{*} u_{0}}(m)$ is well defined for all time. In particular it is at time $t_{0}$, and clearly $\left(m, g, \phi_{t_{0}}^{J^{*} u_{0}}(m)\right)$ is an element of $L$ that projects to $g$ via $p r_{\Gamma}$.

Now we show that $p r_{\Gamma}: p r_{1}^{-1}(m) \rightarrow \mathbf{t}^{-1}(J(m))$ is a covering map again using the path-lifting property of $J$. Given $g$ as above, it is easy to see that we can parametrize a small neighborhood $U^{\mathbf{s}}$ of $g$ in $\mathbf{t}^{-1}(J(m))$ by functions $u$ on $\Gamma_{0}$ (where the $u$ 's lie in the $(n+1)$-dimensional span of coordinate functions centered at $\mathbf{s}(g)$ and a constant function) simply by writing every point in $U^{\mathbf{s}}$ as $\phi_{1}^{\mathbf{s}^{*}(u)}(g)$, the time-1 flow of the integral curve to $X_{\mathbf{s}^{*} u}$ starting at $g$. If $m^{\prime}$ is any point such that $\left(m, g, m^{\prime}\right) \in L$ (so in particular $\left.J\left(m^{\prime}\right)=\mathbf{s}(g)\right)$, denote by $\phi_{1}^{J^{*}(u)}\left(m^{\prime}\right)$ the time-1 flow of the integral curve to $X_{J^{*} u}$ starting at $m^{\prime}$, which is well defined by the completeness of $J$. Then, again because $\mathbf{s}$ and $J$ are Jacobi maps, $\left\{\left(m, \phi_{1}^{\mathbf{s}^{*}(u)}(g), \phi_{1}^{J^{*}(u)}\left(m^{\prime}\right)\right): u \in P\right\}$ is a neighborhood of $\left(m, g, m^{\prime}\right)$ in $p_{1}^{-1}(m)$, and it is clearly mapped diffeomorphically onto $U^{\mathbf{s}}$ by $p r_{\Gamma}$.

Since $p r_{\Gamma}: p r_{1}^{-1}(m) \rightarrow \mathbf{t}^{-1}(J(m))$ is a covering map and $\mathbf{t}^{-1}(J(m))$ is simply connected, we conclude that $p r_{\Gamma}$ is a diffeomorphism.

3.3. $f$-multiplicative functions. Given a free and proper contact groupoid action, we automatically have " $f$-multiplicative functions", which will play an important role in our reduction. So we also call them "reduction functions". 
Proposition-Definition 3.10. If a contact groupoid action of $\Gamma$ on $M$ is free and proper, there exists a non-vanishing function $F$ on $M$ such that

$$
F(m \cdot g)=F(m) f(g) .
$$

We call such a function $f$-multiplicative.

To prove the above we need a technical result about general groupoid actions:

Lemma 3.11. If the action of any Lie groupoid $\Gamma$ on any manifold $M$ is free and proper, then through every point $m \in M$ there exists a disk that meets each $\Gamma$ orbit at most once and transversely.

Proof of Proposition-Definition 3.10. Slices $\left\{D_{i}\right\}$ as in Lemma 3.11 provide manifold charts for the quotient $M / \Gamma$, and the quotient is Hausdorff because the $\Gamma$-action is proper (see Proposition B.8 in [GGK02]). Now choose a subordinate partition of unity, and pull it back to obtain a $\Gamma$-invariant partition of unity $\left\{\left(U_{i}, \rho_{i}\right)\right\}$ on $M$. On each $U_{i}$ construct an $f$-multiplicative function by letting $F_{i}$ be an arbitrary positive function on the slice $D_{i} \subset U_{i}$ and extending $F_{i}$ to $U_{i}$ by $F_{i}(m g)=F_{i}(m) f(g)$. Then

$$
F=\sum \rho_{i} F_{i}
$$

is an $f$-multiplicative function on $M$.

Proof of Lemma 3.11. The proof is analogous to the one of the slice theorem for group actions (see Theorem B.24 in GGK02]). Choose a disk $D$ that intersects the orbit $m \cdot \Gamma$ transversely, and consider the map

$$
\phi: D_{J} \times_{\mathbf{t}} \Gamma \rightarrow M,(u, g) \mapsto u g .
$$

This map is an immersion at $\left(m, 1_{J(m)}\right)$ since the $\Gamma$-action is free at $m$. Here $1_{J(m)}$ denotes $J(m)$ as an element of the space of units.

The above map is injective (one may eventually need to make $D$ smaller), as follows: take sequences $\left(u_{n}, g_{n}\right)$ and $\left(v_{n}, h_{n}\right)$ in $D_{J} \times_{\mathbf{t}} \Gamma$ such that $u_{n}$ and $v_{n}$ converge to $m$ and $u_{n} g_{n}=v_{n} h_{n}$. We may assume that $h_{n} \equiv 1_{J\left(v_{n}\right)}$ (otherwise act by $\left.h_{n}^{-1}\right)$, so $u_{n} g_{n}=v_{n}$. The map $M_{J} \times_{\mathbf{t}} \Gamma \rightarrow M \times M,(m, g) \mapsto(m, m \cdot g)$ is proper because the action is proper, and since the sequence $\left(u_{n}, v_{n}\right)$ converges, the sequence $\left(u_{n}, g_{n}\right)$ also converges, say to $(m, g)$ for some $g \in \Gamma$. Since the action is free, it follows that $g=1_{J(m)}$, and since the map $\phi$ is injective in a neighborhood of $\left(m, 1_{J(m)}\right)$, it follows that the two sequences we started with must agree for $n$ big enough. So the map $\phi$ is injective, and by dimension counting we see that it is a diffeomorphism. Since $\phi$ is $\Gamma$-equivariant and each orbit on the left-hand side of (9) intersects the disk $\left\{\left(u, 1_{J(u)}\right) \mid u \in D\right\}$ exactly once, $D$ is a slice at $m$ for the $\Gamma$-action.

The next two lemmas are technical and are necessary in the proofs of the reduction theorems. In both lemmas we consider a contact groupoid action of a contact groupoid $\Gamma$ on a contact manifold $\left(M, \theta_{M}\right)$ with moment map $J: M \rightarrow \Gamma_{0}$.

Lemma 3.12. For any $f$-multiplicative function $F$ on $M$ and any function $\hat{u}$ constant on the $\Gamma$-orbits we have

$$
d(F \hat{u})\left(E_{M}\right)=0 .
$$


Proof. By equation (6) (choosing $u=1$ there) we know that at any point $m \in M$ we have

$$
0(m) \cdot E_{\Gamma}(x)=E_{M}(m),
$$

where $x=J(m)$. Denoting by $\gamma(\epsilon)$ an integral curve of $E_{\Gamma}$ in $T \mathbf{t}^{-1}(x)$ we have

$$
\begin{aligned}
d F\left(E_{M}(m)\right) & =\left.\frac{d}{d \epsilon}\right|_{0} F(m \cdot \gamma(\epsilon))=\left.\frac{d}{d \epsilon}\right|_{0} F(m) \cdot f(\gamma(\epsilon)) \\
& =F(m) \cdot d f\left(E_{\Gamma}(x)\right)=0,
\end{aligned}
$$

where we used $d f\left(E_{\Gamma}\right)=0$ (see e) in Remark 2.2). The lemma follows since $\hat{u}$ is constant along the $\Gamma$-orbits, and by equation (10) $E_{M}$ is tangent to these orbits.

Lemma 3.13. For any $f$-multiplicative function $F$ and any function $\hat{u}$ constant along the $\Gamma$-orbits, the Hamiltonian vector field $X_{F \hat{u}}$ lies in $T J^{-1}(x)$. In particular $T J^{-1}(x)$ is not contained in $\operatorname{ker}\left(\theta_{M}\right)$ if the action admits an $f$-multiplicative function.

Proof. We will show that

$$
X_{F \hat{u}} \cdot E_{\Gamma}=X_{F \hat{u}}+E_{M},
$$

and the fact that $X_{F \hat{u}}$ and $E_{\Gamma}$ are multipliable implies that $J_{*}\left(X_{F \hat{u}}\right)=t_{*}\left(E_{\Gamma}\right)=0$ as desired. To show (11) we use the same method as in Lemma 3.3 and adapt the notation there, too. We only have to show that $\left(X_{F \hat{u}}, 0, E_{\Gamma}, 0, X_{F \hat{u}}+E_{M}\right)$ lies in $T \mathcal{A}$.

Evaluation of $d \Theta$ on this vector and on $(Y, 0, V, 0, Y \cdot V)$ gives zero, as one can see using $d f\left(E_{\Gamma}\right)=0$, Lemma 3.12 and the $f$-multiplicativity of $F$. Therefore $\left(X_{F \hat{u}}, 0, E_{\Gamma}, 0, X_{F \hat{u}}+E_{M}\right)$ lies in the $d \Theta$-orthogonal to $T \mathcal{A}$. Since evaluation of $\Theta$ on this vector also gives zero and $\mathcal{A}$ is Legendrian by Lemma 3.3, the above vector lies in $T \mathcal{A}$.

\section{Reductions}

In this section, we will first prove the main result using a classical method, i.e. without using groupoid. Then, with a slightly stronger assumption, we can prove the same result with the help of groupoids in a much simpler and illustrative way. Finally, we will establish the relation between the two reductions and explain why they yield the same reduced spaces.

4.1. Classical reduction. We recall that $\Gamma_{x}:=\mathbf{t}^{-1}(x) \cap \mathbf{s}^{-1}(x)$ is the isotropy group of $\Gamma$ at $x$.

Theorem 4.1. Let $\left(\Gamma, \theta_{\Gamma}, f\right)$ act on $\left(M, \theta_{M}\right)$ by a contact groupoid action. Suppose that $x \in \Gamma_{0}$ is a regular value of $J$ and that $\Gamma_{x}$ acts freely and properly on $J^{-1}(x)$, and let $F$ be a $f$-multiplicative function defined on $J^{-1}(x)$. Then the reduced space $M_{x}:=J^{-1}(x) / \Gamma_{x}$ has an induced

(1) contact structure, a representative of which is induced by the restriction of $J^{-1}(x)$ of $8-F^{-1} \theta_{M}$, if $x$ belongs to a contact leaf of the Jacobi manifold $\Gamma_{0}$,

(2) conformal l.c.s. structure, a representative of which is induced by the restriction of $J^{-1}(x)$ of $\left(-F^{-1} d \theta_{M},-F^{-1} d F\right)$, if $x$ belongs to a l.c.s. leaf.

\footnotetext{
${ }^{8}$ The presence of the minus sign here and in Theorem 4.4 will be explained in Example 4.7 below.
} 
Before beginning the proof we need a lemma that involves only the contact groupoid $\left(\Gamma, \theta_{\Gamma}, f\right)$ and not the action:

Lemma 4.2. Consider the isotropy group $\Gamma_{x}$ for some $x \in \Gamma_{0}$. If $x$ lies in a contact leaf, then $\theta_{\Gamma}$ vanishes on vectors tangent to $\Gamma_{x}$. If $x$ lies is an l.c.s. leaf, then $d f$ vanishes on vectors tangent to $\Gamma_{x}$, i.e. $\left.f\right|_{\Gamma_{x}}$ is locally constant.

Proof. Let $g \in \Gamma_{x}$. We will first determine explicitly a basis for $T_{g} \Gamma_{x}=T_{g} \mathbf{t}^{-1}(x) \cap$ $T_{g} \mathbf{s}^{-1}(x)$. To this aim choose functions $\left\{u_{1}, \cdots, u_{n}\right\}$ on $\Gamma_{0}$ vanishing at $x$ such that their differentials at $x$ are linearly independent. We may assume that $\left\{d u_{1}(x), \cdots\right.$, $\left.d u_{\sigma}(x)\right\}$ span $\operatorname{ker}\left(\sharp \Lambda_{0}\right)$. Recall that a basis for $T_{g} \mathbf{t}^{-1}(x)$ is given by $\left\{X_{\mathbf{s}^{*} u_{1}}, \cdots\right.$, $\left.X_{\mathbf{s}^{*} u_{n}}, E_{\Gamma}\right\}$. We have $\mathbf{s}_{*}\left(\sum a_{i} X_{\mathbf{s}^{*} u_{i}}+c E_{\Gamma}\right)=\sum a_{i} \# \Lambda_{0}\left(d u_{i}\right)+c E_{0}$.

If the leaf through $x$ is a contact leaf, then $E_{0}$ does not lie in the image of $\# \Lambda_{0}$, therefore the above vanishes iff $a_{\sigma+1}=\cdots=a_{n}=c=0$. So in this case a basis for $T_{g} \Gamma_{x}$ is

$$
\left\{X_{\mathbf{s}^{*} u_{1}}, \cdots, X_{\mathbf{s}^{*} u_{\sigma}}\right\},
$$

and clearly $\theta_{\Gamma}\left(X_{\mathbf{s}^{*} u_{i}}(g)\right)=u_{i}(x)=0$.

If the leaf through $x$ is a l.c.s. leaf, then $E_{0}$ lies in the image of $\# \Lambda_{0}$, therefore there exists exactly one linear combination $u(x)$ of $u_{\sigma+1}, \cdots, u_{\sigma}$ such that $\# \Lambda_{0}(d u)+E_{0}=0$. So in this case a basis for $T_{g} \Gamma_{x}$ is

$$
\left\{X_{\mathbf{s}^{*} u_{1}}, \cdots, X_{\mathbf{s}^{*} u_{\sigma}}, X_{\mathbf{s}^{*} u}+E_{\Gamma}\right\} .
$$

We have

$$
d f\left(X_{\mathbf{s}^{*} u_{i}}\right)=f(g) d u_{i}\left(E_{0}\right)
$$

using d) and e) in Remark 2.2. So, since for $i=1, \cdots, \sigma$ we have $d u_{i} \in \operatorname{ker}\left(\sharp \Lambda_{0}\right)=$ $\operatorname{Im}\left(\sharp \Lambda_{0}\right)^{\circ}$ and $E_{0} \in \operatorname{Im}\left(\sharp \Lambda_{0}\right)$, we have $d f\left(X_{\mathbf{s}^{*} u_{i}}\right)=0$. Also,

$$
d f\left(X_{\mathbf{s}^{*} u}+E_{\Gamma}\right)=d f\left(X_{\mathbf{s}^{*} u}\right)=f(g) d u\left(E_{0}\right)=f(g) d u\left(-\# \Lambda_{0}(d u)\right)=0 .
$$

Remark 4.3. One can show that $\theta_{\Gamma}$ vanishes on the tangent space of $\Gamma_{x}$ iff $x$ lies in a contact leaf and that $d f$ vanishes there iff $x$ lies in a l.c.s leaf.

Now we are ready to prove Theorem 4.1. We will consider separately the cases when $x$ belongs to a contact or l.c.s. leaf. The steps in the proofs that apply to only one of these two situations are those where Lemma 4.2 is used, i.e. Claim 2 for the contact case and Claims 2 and 4 for the l.c.s. case.

Proof of the contact case. Choose an $f$-multiplicative function $F$ on $J^{-1}(x)$. Such a function always exists (the proof is the same as for Proposition-Definition 3.10). Denote by $\tilde{\theta}_{M}$ the pullback of $\theta_{M}$ to $J^{-1}(x)$. We will show that $-F^{-1} \tilde{\theta}_{M}$ descends to a contact form $\alpha_{F}$ on the reduced space $M_{x}$, and that the corresponding contact structure is independent of the choice of $F$.

Claim 1: $\quad F^{-1} \tilde{\theta}_{M}$ is invariant under the action of $\Gamma_{x}$ on $J^{-1}(x)$.

Proof: Let $Y_{m} \in T_{m} J^{-1}(x)$ and $g \in \Gamma_{x}$. From the definition of contact groupoid action it follows immediately that $\theta_{M}\left(Y_{m} \cdot 0_{g}\right)=f(g) \theta_{M}\left(Y_{m}\right)$. This means that $g^{*}\left(\tilde{\theta}_{M}\right)=f(g) \cdot \tilde{\theta}_{M}$. So

$$
g^{*}\left(F^{-1} \tilde{\theta}_{M}\right)_{m}=F^{-1}(m) f^{-1}(g)\left(g^{*} \tilde{\theta}_{M}\right)_{m}=F^{-1}(m)\left(\tilde{\theta}_{M}\right)_{m}=\left(F^{-1} \tilde{\theta}_{M}\right)_{m} .
$$

Claim 2: The orbits of the $\Gamma_{x}$-action are tangent to the kernel of $\tilde{\theta}_{M}$. 
Proof: To see this, let $m \in J^{-1}(x)$ and let $V_{x} \in T_{x} \Gamma_{x}$. Again from the definition of contact groupoid action we infer that $\theta_{M}\left(0_{m} \cdot V_{x}\right)=\theta_{\Gamma}\left(V_{x}\right)$, which vanishes by Lemma 4.2

Claim 3: $-F^{-1} \tilde{\theta}_{M}$ descends to a contact form $\alpha_{F}$ on $J^{-1}(x) / \Gamma_{x}$.

Proof: It is clear by the above two claims that $-F^{-1} \tilde{\theta}_{M}$ descends, so we only have to ensure that it gives rise to a contact form. To this aim we first extend $F$ arbitrarily to an open neighborhood of $J^{-1}(x)$ in $M$ and we determine explicitly $\operatorname{ker}\left(d\left(F^{-1} \tilde{\theta}_{M}\right)\right)$, i.e. $T_{m} J^{-1}(x) \cap T_{m} J^{-1}(x)^{d\left(F^{-1} \theta_{M}\right)}$. Note that

$$
d\left(F^{-1} \theta_{M}\right)\left(X_{J^{*} u}, X\right)=F^{-2} d u_{x}\left(J_{*} X_{F}\right) \theta_{M}(X)-F^{-1} d u_{x}\left(J_{*} X\right)+F^{-2} d F(X) J^{*} u .
$$

This together with the fact that $X_{F}$ is the Reeb vector field of $F^{-1} \theta_{M}$ implies that

$$
T_{m} J^{-1}(x)^{d\left(F^{-1} \theta_{M}\right)} \supset\left\{X_{J^{*} u} \mid u(x)=0, d u_{x}\left(J_{*} X_{F}\right)=0\right\} \oplus X_{F}
$$

and

$$
\left\{X_{J^{*} u} \mid u(x)=0, d u_{x}\left(J_{*} X_{F}\right)=0\right\}^{d\left(F^{-1} \theta_{M}\right)} \subset T_{m} J^{-1}(x)+X_{F} .
$$

Since $\operatorname{ker}\left(d F^{-1} \theta_{M}\right)=\operatorname{span}\left\{X_{F}\right\}$, by taking the orthogonals with respect to $d F^{-1} \theta_{M}$ on both sides of the above two equations, we obtain the opposite inclusions. Therefore we actually have equality in (13) and (14).

By Lemma 4.2 and (6), and the fact that $d\left(F^{-1} \theta_{M}\right)$ descends, we have

$$
\begin{aligned}
\left\{X_{J^{*} u} \mid u(x)=0, d u_{x} \in \operatorname{ker}\left(\sharp \Lambda_{0}\right)\right\}=0_{m} \cdot T_{x} \Gamma_{x} & \subset \operatorname{ker} d\left(F^{-1} \tilde{\theta}_{M}\right) \\
& \subset T_{m} J^{-1}(x)^{d\left(F^{-1} \theta_{M}\right) .} .
\end{aligned}
$$

Combining with (13), this says that if $u(x)=0$ and $d u_{x} \in \operatorname{ker}\left(\sharp \Lambda_{0}\right)=\operatorname{im}\left(\sharp \Lambda_{0}\right)^{0}$ (the annihilator of the image of $\left.\sharp \Lambda_{0}\right)$, then $d u_{x}\left(J_{*} X_{F}\right)=0$. This means that $J_{*} X_{F} \in \operatorname{im}\left(\sharp \Lambda_{0}\right) 9$ Therefore there exists some function $u_{0}$ vanishing at $x$ such that $J_{*} X_{F}(m)=\left(\sharp \Lambda_{0} d u_{0}\right)(x)$. Since $X_{F-J^{*} u_{0}}$ lies in $T_{m} J^{-1}(x)$ but not in $\operatorname{ker}\left(\theta_{M}\right)$ we conclude that $T_{m} J^{-1}(x) \not \subset \operatorname{ker}\left(\theta_{M}\right)_{m}$.

Now set $J_{*}\left(X_{J^{*} u}+c X_{F}\right)=\sharp \Lambda_{0} d u+c J_{*} X_{F}$ equal to zero. By (13) we conclude that

$$
\operatorname{ker}\left(d\left(F^{-1} \tilde{\theta}_{M}\right)\right)=T_{m} J^{-1}(x) \cap T_{m} J^{-1}(x)^{d\left(F^{-1} \theta_{M}\right)}=0_{m} \cdot T_{x} \Gamma_{x} \oplus\left(X_{F}-X_{J^{*} v}\right),
$$

where $v$ is the unique function vanishing at $x$ (could be 0 ) on $\Gamma_{0}$ such that $\sharp \Lambda_{0} d v_{x}=$ $J_{*} X_{F}$. Uniqueness and existence are ensured by the facts that $T_{m} J^{-1}(x) \not \subset$ $\operatorname{ker}\left(\theta_{M}\right)_{m}$ and $J_{*} X_{F} \in \operatorname{im}\left(\sharp \Lambda_{0}\right)$. Therefore $d \alpha_{F}$ induced on $M_{x}$ by $F^{-1} \tilde{\theta}_{M}$ has one-dimensional kernel spanned by the image of $X_{F}-X_{J^{*} v}$, and since

$$
F^{-1} \tilde{\theta}_{M}\left(X_{F}-X_{J^{*} v}\right)=1 \neq 0
$$

it follows that $\alpha_{F}$ is a contact form.

Claim 4: The contact structure on $M_{x}$ given by $\operatorname{ker}\left(\alpha_{F}\right)$ is independent of the chosen $f$-multiplicative function $F$.

Proof: From the construction of the contact form $\alpha_{F}$, it is easy to see that, for another $f$-multiplicative function $\hat{F}$ on $J^{-1}(x)$,

$$
\pi^{*}\left(\alpha_{F}\right)=\frac{\hat{F}}{F} \cdot \pi^{*}\left(\alpha_{\hat{F}}\right),
$$

\footnotetext{
${ }^{9}$ Notice that in Lemma 3.13 we showed that if $F$ is $f$-multiplicative on the whole of $M$, then $J_{*} X_{F}=0$.
} 
where $\pi: J^{-1}(x) \rightarrow M_{x}$ is the projection. By the $f$-multiplicativity, $\frac{\hat{F}}{F}$ is $\Gamma_{x^{-}}$ invariant, so it descends to a function $Q$ on $M_{x}$. Since $\pi^{*}$ is injective, we have $\alpha_{F}=Q \alpha_{\hat{F}}$.

Now we prove the locally conformal symplectic case:

Proof of the l.c.s. case. Adapt the same notation as above. We will show that the two-form $-F^{-1} d \tilde{\theta}_{M}$ and the one-form $-F^{-1} d F$ descend to forms $\Omega_{F}$ and $\omega_{F}$ respectively on $M_{x}$. The reduced space $M_{x}$ together with the pair $\left(\Omega_{F}, \omega_{F}\right)$ will be a l.c.s. manifold, i.e. $\Omega_{F}$ is non-degenerate, $\omega_{F}$ closed, and $d \Omega_{F}=\omega_{F} \wedge \Omega_{F}$. Furthermore, a different choice of $f$-multiplicative function will give a conformally equivalent 1.c.s. structure on $M_{x}$.

Claim 1: $F^{-1} d \tilde{\theta}_{M}$ is invariant under the $\Gamma_{x}$ action on $J^{-1}(x)$.

Proof: Let $g \in \Gamma_{x}$ and $m \in J^{-1}(x)$. Notice that $g^{*}\left(\tilde{\theta}_{M}\right)=f(g) \cdot \tilde{\theta}_{M}$, hence $g^{*}\left(d \tilde{\theta}_{M}\right)=f(g) \cdot d \tilde{\theta}_{M}$. A calculation analogous to the one presented in Claim 1 of the proof of the contact case allows us to conclude that $g^{*}\left(F^{-1} d \tilde{\theta}_{M}\right)=F^{-1} d \tilde{\theta}_{M}$. $\nabla$

Claim 2: $-F^{-1} d \tilde{\theta}_{M}$ descends to a non-degenerate two-form $\Omega_{F}$ on $M_{x}$.

Proof: Since $-F^{-1} d \tilde{\theta}_{M}$ is a non-vanishing multiple of $d \tilde{\theta}_{M}$, the above claim will be true if and only if at all $m \in J^{-1}(x)$

$$
0_{m} \cdot T_{x} \Gamma_{x}=\operatorname{ker}\left(d \tilde{\theta}_{M}\right)\left(=T_{m} J^{-1}(x) \cap\left(T_{m} J^{-1}(x)\right)^{d \theta_{M}}\right) .
$$

For the inclusion " $\subset$ " we compute for any $V \in T_{x} \Gamma_{x}$ and $Y \in T_{m} J^{-1}(x)$ that $d \theta_{M}\left(0_{m} \cdot V, Y\right)=0$ by taking the exterior derivative of (4) in Definition 3.1 and using Lemma 4.2. So $0_{m} \cdot V \in T_{m}\left(J^{-1}(x)\right)^{d \theta_{M}}$, and since $\Gamma_{x}$ acts on $J^{-1}(x)$ the first inclusion is proven.

For the opposite inclusion " $\supset$ " we will show below that

$$
0_{m} \cdot T_{x} \mathbf{t}^{-1}(x)=\left(T_{m} J^{-1}(x) \cap \mathcal{H}_{m}\right)^{d \theta_{M}},
$$

where $\mathcal{H}_{m}$ denotes the kernel of $\left(\theta_{M}\right)_{m}$. Then, taking the $d \theta_{M}$-complement of the relation $T_{m} J^{-1}(x) \cap \mathcal{H}_{m} \subset T_{m} J^{-1}(x)$, we obtain

$$
0_{m} \cdot T_{x} \mathbf{t}^{-1}(x) \supset\left(T_{m} J^{-1}(x)\right)^{d \theta_{M}} .
$$

Clearly we preserve the inclusion if we intersect both sides with $T_{m} J^{-1}(x)$. Now, since for any $V \in T_{x} \mathbf{t}^{-1}(x)$ we have $0_{m} \cdot V \in T_{m} J^{-1}(x) \Leftrightarrow V \in T_{x} \mathbf{s}^{-1}(x)$, we obtain

$$
0_{m} \cdot T_{x} \Gamma_{x}=0_{m} \cdot T_{x} \mathbf{t}^{-1}(x) \cap T_{m} J^{-1}(x) \supset T_{m} J^{-1}(x) \cap\left(T_{m} J^{-1}(x)\right)^{d \theta_{M}}
$$

and we are done.

To complete the proof of " $\supset$ " we still have to show equation (15). By d) in Remark 2.2 and (6), we have $0_{m} \cdot T_{x} \mathbf{t}^{-1}(x)=0_{m} \cdot\left\{X_{\mathbf{s}^{*} u}(x)\right\}=\left\{X_{J^{*} u}(m)\right\}$, where $u$ ranges over all functions on $\Gamma_{0}$. Notice that for $Y \in \mathcal{H}_{m}$ we have $d \theta_{M}\left(X_{J^{*} u}, Y\right)=$ $-d u\left(J_{*} Y\right)$, so that

$$
\left\{X_{J^{*} u}(m)\right\}^{d \theta_{M}} \cap \mathcal{H}_{m}=T_{m} J^{-1}(x) \cap \mathcal{H}_{m} .
$$

Since the Reeb vector field $E_{M}$ lies in $\left\{X_{J^{*} u}\right\}$, taking orthogonals of the above, we are done.

Claim 3: $F^{-1} d F$ is invariant under the $\Gamma_{x}$ action on $J^{-1}(x)$.

Proof: The $f$-multiplicativity of $F$ implies $\left(g^{*} d F\right)=f(g) \cdot d F$. The rest of the proof is analogous to the one of Claim 1 of the proof of the contact case.

Claim 4: $-F^{-1} d F$ descends to a one-form $\omega_{F}$ on $M_{x}$. 
Proof: We have to check that if $V \in T_{x} \Gamma_{x}$ then $0_{m} \cdot V$ lies in the kernel of $-F^{-1} d F$. This is satisfied because $d F\left(0_{m} \cdot V\right)=F(m) d f(V)=0$ by the $f$ multiplicativity of $F$ and by the second part of Lemma 4.2 .

Claim 5: The two-form $\Omega_{F}$ induced by $-F^{-1} d \tilde{\theta}_{M}$ and the one-form $\omega_{F}$ induced by $-F^{-1} d F$ endow $M_{x}$ with a l.c.s. structure.

Proof: We have to show that $\omega_{F}$ is closed and that $d \Omega_{F}=\omega_{F} \wedge \Omega_{F}$. Since $\pi: J^{-1}(x) \rightarrow J^{-1}(x) / \Gamma_{x}$ is a submersion, it suffices to show $\pi^{*}\left(d \omega_{F}\right)=0$ and $\pi^{*} d \Omega_{F}=\pi^{*}\left(\omega_{F} \wedge \Omega_{F}\right)$. The former is clear since $\pi^{*} \omega_{F}=-d(\ln |F|)$ is exact, the latter follows by a short computation.

Claim 6: The conformal class of the l.c.s. structure on $M_{x}$ given by $\omega_{F}$ and $\Omega_{F}$ is independent of the choice of $F$.

Proof: Let $\hat{F}$ be another $f$-multiplicative function on $J^{-1}(x)$ and denote by $Q$ the function on $M_{x}$ induced by $\frac{\hat{F}}{F}$. We have $\Omega_{F}=Q \Omega_{\hat{F}}$ because

$$
\pi^{*} \Omega_{F}=-F^{-1} d \tilde{\theta}_{M}=-\frac{\hat{F}}{F} \hat{F}^{-1} d \theta_{M}=\pi^{*}\left(Q \cdot \Omega_{\hat{F}}\right),
$$

and similarly we obtain $\omega_{F}=d(\ln |Q|)+\omega_{\hat{F}}$. Now a standard computation shows that the identity $I d:\left(M_{x}, \Omega_{F}, \omega_{F}\right) \rightarrow\left(M_{x}, \Omega_{\hat{F}}, \omega_{\hat{F}}\right)$ is a $Q$-conformal Jacobi map. $\nabla$

4.2. Global reduction. In this subsection, we will achieve the desired reduction result through a global reduction procedure. It is technically easier and also suggests that the reduced spaces "glue well together".

The key observation (see [MW88]) is the following: if a contact groupoid $\Gamma$ acts (say from the right) on a manifold $M$ with moment map $J$, then the orbit space of the action is

$$
M / \Gamma=\coprod_{\mathcal{O}} J^{-1}(\mathcal{O}) / \Gamma
$$

where the disjoint union ranges over all orbits $\mathcal{O}$ of the groupoid $\Gamma$, i.e. over all leaves of the Jacobi manifold $\Gamma_{0}$.

Also, for each $x \in \mathcal{O}$, by the equivariance of $J$ we have

$$
J^{-1}(x) / \Gamma_{x}=J^{-1}(\mathcal{O}) / \Gamma .
$$

So topologically $M / \Gamma$ is equal to a disjoint union of reduced spaces, one for each leaf $\mathcal{O}$ of $\Gamma_{0}$. This suggests that the reduced space is a Jacobi manifold with foliation given by these individual reduced spaces. Indeed we have:

Theorem 4.4. Let $\left(\Gamma, \theta_{\Gamma}, f\right)$ act on $\left(M, \theta_{M}\right)$ freely and properly, and let $F$ be an $f$ multiplicative function on $M$. Then there is an induced Jacobi structure on $M / \Gamma$ such that the projection $\mathrm{pr}: M \rightarrow M / \Gamma$ is a $-F$-conformal Jacobi map 10

Moreover, the Jacobi foliation is given exactly by (the connected components of) the decomposition

$$
M / \Gamma=\coprod_{\mathcal{O}, x \in \mathcal{O}} J^{-1}(x) / \Gamma_{x},
$$

and the reduced manifolds $J^{-1}(x) / \Gamma_{x}$ are contact or l.c.s. manifolds exactly when the leaves $\mathcal{O}$ through $x$ are.

The conformal class of the Jacobi structure on $M / \Gamma$ is independent of the choice of $F$.

\footnotetext{
${ }^{10}$ The presence of the minus sign here will be explained in Example 4.7 below.
} 
We first determine that the $\Gamma$-action on $M$ preserves the contact form up to a factor of $f$ :

Lemma 4.5. Let $\Sigma$ be a Legendrian bisection of $\left(\Gamma, f, \theta_{\Gamma}\right)$ and $r_{\Sigma}: M \rightarrow M$, $m \mapsto m \cdot \Sigma(J(m))$ the induced diffeomorphism of $M$, where $\Sigma$ is viewed as a section of $\mathbf{t}$. Then

$$
r_{\Sigma}^{*} \theta_{M}=f(\Sigma \circ J) \cdot \theta_{M} .
$$

Furthermore, through any given point of $\Gamma$ there exists a local Legendrian bisection.

Proof. Let $m \in M, V \in T_{m} M, g:=\Sigma(J(m))$ and $Y:=\Sigma_{*} J_{*} V \in T_{g} \Gamma$. Then since $Y$ is tangent to a Legendrian bisection

$$
r_{\Sigma}^{*} \theta_{M}(V)=\theta_{M}(V \cdot Y)=f(g) \cdot \theta_{M}(V)+\theta_{\Gamma}(Y)=f(g) \cdot \theta_{M}(V) .
$$

This establishes the first part of the lemma.

Now we show that there exists a local Legendrian bisection of $\Gamma$ through every $g \in \Gamma$. By a generalized Darboux theorem we can assume that a neighborhood of $g$ in $\left(\Gamma, \theta_{\Gamma}\right)$ is equal to a neighborhood of the origin in $\left(\mathbb{R}^{2 n+1}, d z-\sum x_{i} d y_{i}\right)$. Consider the natural projection $\mathbb{R}^{2 n+1} \rightarrow \mathbb{R}^{2 n}$ with kernel the $z$-axis. By [Daz97, the $(n+1)$-dimensional subspaces $T_{g} \mathbf{s}^{-1}$ and $T_{g} \mathbf{t}^{-1}$ are both not contained in $\operatorname{ker}\left(\theta_{M}\right)_{g}$, so the derivative at the origin $(=g)$ of the above projection maps $T_{g} \mathbf{s}^{-1} \cap$ $\operatorname{ker}\left(\theta_{M}\right)_{g}$ and $T_{g} \mathbf{t}^{-1} \cap \operatorname{ker}\left(\theta_{M}\right)_{g}$ to subspaces of $\mathbb{R}^{2 n}$ of dimension $n$. Therefore we can find a Lagrangian subspace of $\mathbb{R}^{2 n}$ which is transversal to both. It is known (see SW99, p. 186) that any Lagrangian submanifold of $\mathbb{R}^{2 n}$ through the origin which is exact (this condition is always satisfied locally) can be lifted to a Legendrian submanifold of $\mathbb{R}^{2 n+1}$ through the origin. The lift of this Lagrangian subspace will be a Legendrian bisection nearby $g$, because it will be transversal to both $T_{g} \mathbf{s}^{-1}$ and $T_{g} \mathbf{t}^{-1}$.

Proof of Theorem 4.4. We fix an $f$-multiplicative function $F$. It follows from Lemma 4.5 that for any Legendrian bisection $\Sigma$ the induced map $r_{\Sigma}$ on $M$ preserves $-F^{-1} \theta_{M}$, which corresponds to the Jacobi structure on $M$ obtained by $-F$ conformal change of the original one 11 Therefore $r_{\Sigma}$ preserves the corresponding Jacobi bracket $\{\cdot, \cdot\}_{-F}=-F^{-1}\{-F \cdot,-F \cdot\}$, and for any functions $\hat{h}$ and $\hat{k}$ on $M$ which are constant along the $\Gamma$-orbits we have

$$
r_{\Sigma}^{*}\{\hat{h}, \hat{k}\}_{-F}=\left\{r_{\Sigma}^{*} \hat{h}, r_{\Sigma}^{*} \hat{k}\right\}_{-F}=\{\hat{h}, \hat{k}\}_{-F} .
$$

So, by the existence of local Legendrian bisections in Lemma 4.5 $\{\hat{h}, \hat{k}\}_{-F}$ is also a function constant along the orbits. Hence such functions are closed under the new bracket $\{\cdot, \cdot\}_{-F}$.

By Lemma $3.11 M / \Gamma$ is a manifold. The bracket $\{\cdot, \cdot\}_{-F}$ induces a bracket on $C^{\infty}(M / \Gamma)$ : for any functions $h, k$ on $M / \Gamma$ we define

$$
\{h, k\}_{M / \Gamma}=\left\{p r^{*} h, p r^{*} k\right\}_{-F} .
$$

The induced bracket still satisfies the Jacobi identity and (2). That is, $C^{\infty}(M / \Gamma)$ is endowed with a structure of local Lie algebra in the sense of Kirillov, therefore $M / \Gamma$ is endowed with the structure of a Jacobi manifold with Jacobi bracket $\{\cdot, \cdot\}_{M / \Gamma}$ (see Daz97, p. 434). The map $p r: M \rightarrow M / \Gamma$ is $-F$-conformal Jacobi by construction.

\footnotetext{
11 This follows from the general fact that if $(N, \theta)$ is any contact manifold and $\varphi$ a non-vanishing function on $N$, then the Jacobi structure corresponding to $\varphi \theta$ is $\left(\varphi^{-1} \Lambda, X_{\varphi^{-1}}\right)$.
} 
Now we will show that for $x \in \Gamma_{0}$ (any connected component of) $J^{-1}(x) / \Gamma$ is a leaf of $M / \Gamma$, i.e. that $\operatorname{span}_{h \in C^{\infty}(M / \Gamma)}\left\{X_{h}\right\}=T\left(J^{-1}(x) / \Gamma\right)$. It is enough to show that at any $m \in J^{-1}(x)$

$$
\operatorname{span}_{\{\hat{h} \text { is } \Gamma \text {-invariant }\}}\left\{X_{\hat{h}}^{-F}(m)\right\}=T_{m} J^{-1}(x),
$$

since $\left.p r\right|_{J^{-1}(x)}: J^{-1}(x) \longrightarrow J^{-1}(x) / \Gamma$ is a submersion and for any $\Gamma$-invariant function $\hat{h}=p r^{*}(h)$ we have $p r_{*}\left(X_{\hat{h}}^{-F}\right)=X_{h}$. Here $X^{-F}$ denotes the Hamiltonian vector field with respect to the new $-F$-twisted Jacobi structure on $M$.

The inclusion " $\subset$ " in equation (16) is clearly implied by Lemma 3.13.

The inclusion " $\supset$ " can be seen by a simple dimension counting. Suppose $\operatorname{dim} M=$ $k$ and $\operatorname{dim} \Gamma=2 n+1$. Since the action is free, each $\Gamma$-orbit has dimension $n+1$, so the space $\left\{d \hat{h}_{m}\right\}$ has dimension $k-n-1$. Choose a basis $\left\{d \hat{h}_{1}, \ldots, d \hat{h}_{k-n-1}\right\}$ of this space, where the $\hat{h}_{i}$ 's are functions vanishing at $m$. The corresponding vectors $X_{\hat{h}_{i}}^{-F}(m)$ are linearly independent, because none of them lies in $\operatorname{ker}\left(-\sharp F \Lambda_{M}\right)=$ $\operatorname{span}\left\{\theta_{M}\right\}$ (this is true since each $d \hat{h}_{i}$ annihilates $E_{M}$ by equation (6) but $\theta_{M}$ does not). Adding $X_{1}^{-F}(m)$ we obtain a basis for $\left\{X_{\hat{h}}^{-F}(m)\right\}$ consisting of $k-n$ elements. Since by Lemma $3.5 J$ is a submersion, $\operatorname{dim} J^{-1}(x)$ is also $k-n$, so (16) is proven.

A similar dimension counting shows that the reduced manifold $J^{-1}(x) / \Gamma_{x}$ is a contact (l.c.s.) manifolds exactly when the leaf $\mathcal{O}$ through $x$ is: $J^{-1}(\mathcal{O}) / \Gamma$ has dimension $k-2 n-1+\operatorname{dim}(\mathcal{O})$, which has the same parity as $\operatorname{dim}(\mathcal{O})$ because $k$ is always odd.

If we take another $f$-multiplicative function $G$, then $\frac{G}{F}$ is constant along the orbits, therefore it defines a function $Q$ on $M / \Gamma$. It is easy to see that the bracket on $M / \Gamma$ induced by $\{\cdot, \cdot\}_{-G}$ is given by a $Q$-conformal change of the bracket induced by $\{\cdot, \cdot\}-F$.

Remark 4.6. It turns out that the global reduction can be carried out via symplectification, namely, one can go to the symplectification of the contact groupoid and use reduction via symplectic groupoids in the sense of [MW88. However it is not obvious how to carry out the pointwise reduction (which requires weaker assumptions) using symplectification.

Example 4.7 (Groupoid multiplication). If $\left(M, \theta_{M}\right)=\left(\Gamma, \theta_{\Gamma}\right)$ and the action $\Phi$ is by right multiplication ( $s o J=\mathbf{s}$ ), then the map $\mathbf{t}: M \rightarrow \Gamma_{0}$ gives an identification $M / \Gamma \cong \Gamma_{0}$. Under this identification the map $p r: M \rightarrow M / \Gamma$ corresponds exactly to t. Endow $M / \Gamma \cong \Gamma_{0}$ with the Jacobi structure as by Theorem 4.4 using the function $F:=f$. Since $\mathbf{t}$ is a $-f$-Jacobi map for the original Jacobi structure on $\Gamma_{0}$, the induced Jacobi structure on $\Gamma_{0}$ is exactly the original one.

4.3. Relation between the two reductions. Next we show that the classical reduction procedure (Theorem 4.1) and the groupoid reduction procedure (Theorem 4.4) both yield the same contact or l.c.s. structures on the reduced spaces $J^{-1}(x) / \Gamma_{x}$. It is enough to show:

Theorem 4.8. Let $\left(\Gamma, \theta_{\Gamma}, f\right)$ act on $\left(M, \theta_{M}\right)$ by a contact groupoid action freely and properly. Choose an $f$-multiplicative function $F$ and endow $M / \Gamma$ with a Jacobi structure as in Theorem 4.4. Then the contact or l.c.s. structures on $M_{x}:=$ $J^{-1}(x) / \Gamma_{x}$ are induced by the restrictions to $J^{-1}(x)$ of the following forms. 
(1) $-F^{-1} \theta_{M}$ if $M_{x}$ is a contact leaf,

(2) $\left(-F^{-1} d \theta_{M},-F^{-1} d F\right)$ if $M_{x}$ is a l.c.s. leaf.

Proof. Case 1: $M_{x}$ is a contact leaf. Denote by $\alpha_{F}$ the contact form on $J^{-1}(x) / \Gamma_{x}$ given by the Jacobi structure on $M / \Gamma$. We consider $\left.p r\right|_{J^{-1}(x)}: J^{-1}(x) \rightarrow J^{-1}(x) / \Gamma_{x}$ and want to show that at $m \in J^{-1}(x)$ we have $\left(\left.p r\right|_{J^{-1}(x)}\right)^{*} \alpha_{F}=-F^{-1} \tilde{\theta}_{M}$, where $\tilde{\theta}_{M}$ denotes the restriction of $\theta_{M}$ to $J^{-1}(x)$. By equation (16) and $p r_{*}\left(X_{p r^{*} h}^{-F}\right)=X_{h}$, we only have to show that

$$
\alpha_{F}\left(X_{h}\right)=-F^{-1} \tilde{\theta}_{M}\left(X_{p r^{*} h}^{-F}\right),
$$

which is obvious since both sides are equal to $h(x)$.

Case 2: $M_{x}$ is an l.c.s. leaf. Denote by $\omega_{F}$ and $\Omega_{F}$ the one-form and twoform defining the l.c.s. structure on $J^{-1}(x) / \Gamma_{x}$. As above we want to show that $\left(\left.p r\right|_{J^{-1}(x)}\right)^{*} \omega_{F}=-F^{-1} d F$ and $\left(\left.p r\right|_{J^{-1}(x)}\right)^{*} \Omega_{F}=-F^{-1} d \tilde{\theta}_{M}$. A computation using $d F\left(E_{M}\right)=0$ (by Lemma 3.12) and $d \hat{h}\left(E_{M}\right)=0$ (since $E_{M}$ is tangent to the $\Gamma$-orbits by equation (6) ) shows that for all $h \in C^{\infty}(M / \Gamma)$ we have

$$
\omega_{F}\left(X_{h}\right)=d h\left(E_{0}\right)=-F^{-1} d F\left(X_{p r^{*} h}^{-F}\right)
$$

and

$$
\begin{aligned}
\Omega_{F}\left(X_{h}, X_{k}\right) & =-k \cdot d h\left(E_{0}\right)+h \cdot d k\left(E_{0}\right)-d h\left(\# \Lambda_{0} d k\right) \\
& =-F^{-1} d \tilde{\theta}_{M}\left(X_{p r^{*} h}^{-F}, X_{p r^{*} k}^{-F}\right),
\end{aligned}
$$

so we are done.

\section{RELATION With OTHER CONTACT REDUCTIONS AND PREQUANTIZATION}

In this section, which can be read independently of the previous ones, we clarify Willett's procedure for contact reduction and point out the relation between the reduced spaces by contact groupoid reduction on one hand and Willett's and Albert's reduced spaces on the other hand.

5.1. Relation with Willett's reduction. Suppose $G$ is a Lie group acting on a contact manifold $\left(M, \theta_{M}\right)$ from the right preserving the contact one form $\theta_{M}$. A moment map [Alb89], Wil02] is a map $\phi$ from the manifold $M$ to $\mathfrak{g}^{*}$ (the dual of the Lie algebra) such that for all $v$ in the Lie algebra $\mathfrak{g}$,

$$
\langle\phi, v\rangle=\theta_{M}\left(v_{M}\right),
$$

where $v_{M}$ is the infinitesimal generator of the action on $M$ given by $v$. The moment map $\phi$ is automatically equivariant with respect to the (right) coadjoint action of $G$ on $\mathfrak{g}^{*}$ given by $\xi \cdot g=L_{g}^{*} R_{g^{-1}}^{*} \xi$. A group action as above together with its moment map is called Hamiltonian action. In [Wil02], Willett defines the contact reduction at the point $\xi \in \mathfrak{g}^{*}$ to be

$$
M_{\xi}^{W}:=\phi^{-1}\left(\mathbb{R}^{+} \cdot \xi\right) / K_{\xi},
$$

where $K_{\xi}$ is the unique connected subgroup of $G_{\xi}$ (the stabilizer group at $\xi$ of the coadjoint action) such that its Lie algebra is the intersection of ker $\xi$ and $\mathfrak{g}_{\xi}$ (the Lie algebra of $G_{\xi}$ ). If the following three conditions hold:

a) $\operatorname{ker} \xi+\mathfrak{g}_{\xi}=\mathfrak{g}$,

b) $\phi$ is transverse to $\mathbb{R}^{+} \cdot \xi$,

c) the $K_{\xi}$ action is proper, 
then the reduced space $M_{\xi}^{W}$ is a contact orbifold. It is a manifold if the $K_{\xi}$ action is free. When $\xi=0$, Willett's reduced space is the same as the one obtained by Albert Alb89.

It turns out that Willett's reduction is strongly related to (the prequantization of) our reduction.

First of all, given a contact Hamiltonian action, we naturally have a groupoid action. Using the notation of Example 2.3. we have

Proposition 5.1. Identify $S^{*} G$ and $S\left(\mathfrak{g}^{*}\right) \rtimes G$ by right translation. Then a Hamiltonian $G$ action on $\left(M, \theta_{M}\right)$ gives rise to a contact groupoid action of $S^{*} G$ on $\left(M, \frac{\theta_{M}}{\|\phi\|}\right)$ by

$$
m \cdot([\xi], g):=m \cdot g
$$

with moment map $J=[\phi]$, if 0 is not in the image of $\phi$. Here $[\cdot]$ denotes the equivalence class under the $\mathbb{R}^{+}$action.

Proof. Let $m$ be in $M$ and let $([\xi], g)$ be in $S\left(\mathfrak{g}^{*}\right) \rtimes G$ with $J(m)=\mathbf{t}([\xi], g)=[\xi]$. Since the coadjoint action on $\mathfrak{g}^{*}$ is linear and using the equivariance of $\phi$, one can easily check that the given action is a groupoid action (Definition 3.1).

To see whether this is a contact groupoid action, we only have to verify (4). Suppose $\left(Y,\left(\delta \xi, R_{g_{*}} v\right)\right) \in T_{(m,(\xi, g))}\left(M_{J} \times_{\mathbf{t}} \mathfrak{g}^{*} \rtimes G\right)$, where $v$ is an element in $\mathfrak{g}$ and $R_{g}$ denotes right translation by $g$. Notice that the image of $\left(Y, R_{g_{*}} v\right)$ under the derivative of the group action map $M \times G \rightarrow M$ is $\left(v_{M}+Y\right) \cdot g$. Here by $\cdot g$ we denote the lift action of $G$ on $T M$. Then (4) follows from (17).

Remark 5.2. If we are given a free Hamiltonian contact action, from this claim, we can see that we can perform our reduction at every point except for 0 . For $\xi=0$, one can use another groupoid (see Proposition 5.11) to make up this deficiency.

Now we give another characterization of the conditions a), b) and c) above which ensure that Willett's reduced space be a contact orbifold.

Lemma 5.3. Given a free Hamiltonian action of a compact group $G$ on a contact manifold $M$, Willett's conditions for contact reduction a), b) and c) are equivalent to the following two conditions:

(1) $[\xi]$ is a regular value of $J$;

(2) $\xi$ is conjugate to a multiple of an integer point.

For any Lie algebra $\mathfrak{t}$ of a maximal torus in $G$ we call a point of $\mathfrak{t}^{*}$ integer if it has integral pairing with all elements of $\operatorname{ker}\left(\left.\exp \right|_{\mathfrak{t}}\right)$.

Proof. We identify $\mathfrak{g}$ and $\mathfrak{g}^{*}, \mathfrak{t}$ and $\mathfrak{t}^{*}$ using a bi-invariant metric on $G$, where $\mathfrak{t}$ is the Lie algebra of a maximal torus $T$ of $G$. We may assume $\xi$ is inside $\mathfrak{t}$ since the statement is invariant under coadjoint actions. Then condition a) is automatically satisfied, since regarding $\xi$ as an element of $\mathfrak{g}$ we have ker $\xi=\xi^{\perp}$. Clearly, (1) is equivalent to the transversality condition b). So we only have to show that (2) is equivalent to condition c).

In general, if a compact group $G$ acts on a manifold $N$, then the induced action of a subgroup $K$ is proper if and only if $K$ is also compact. This can be easily seen through the definition of properness (cf. (5)): an action $\Phi$ of $K$ on $N$ is proper iff the map $\Phi \times i d: K \times N \rightarrow N \times N$ is proper. Let $O$ be an orbit of the action of $G$ on $N$. Then the compactness of $O$ implies the compactness of 
$(\Phi \times i d)^{-1}(O \times O)=K \times O$, hence of $K$. In particular, applying this to our case, we see that c) is equivalent to $K_{\xi}$ being compact.

Notice that the Lie algebra of $G_{\xi}$ is $\mathfrak{g}_{\xi}=\{a:[a, \xi]=0\}$ and the Lie algebra of $K_{\xi}$ is $\mathfrak{k}_{\xi}=\xi^{\perp} \cap \mathfrak{g}_{\xi}$. So we have $\mathfrak{g}_{\xi}=\mathfrak{k}_{\xi} \oplus \xi \cdot \mathbb{R}$.

If $\xi$ is not a multiple of any integer point, $\mathfrak{k}_{\xi}$ will contain a vector whose coordinates are linearly independent over $\mathbb{Z}$, hence the Lie algebra of an irrational flow. This is not hard to see because the set of vectors with $\mathbb{Z}$-linearly dependent coordinates is the union of countably many hyperplanes indexed by $\mathbb{Z}^{n}$ and $\mathfrak{k}_{\xi}$ is not one of these, so the vectors of $\mathfrak{k}_{\xi}$ with $\mathbb{Z}$-linearly dependentent coordinates are contained in countably many hyperplanes of $\mathfrak{k}_{\xi}$. The fact that this vector has $\mathbb{Z}$-linearly independent coordinates exactly means that it is not contained in any subtorus. So the Lie group $K_{\xi} \cap T$ integrating $\mathfrak{k}_{\xi} \cap \mathfrak{t}$ is dense in $T$. If $K_{\xi}$ is compact, then $K_{\xi} \cap T$ is compact too; hence $K_{\xi} \cap T=T$. But this is impossible because its Lie algebra $\mathfrak{k}_{\xi} \cap \mathfrak{t}$ does not contain $\xi$.

On the other hand, if $\xi$ is a multiple of some integer point, then the Lie group $K_{\xi} \cap T$ integrating $\mathfrak{k}_{\xi} \cap \mathfrak{t}$ is compact. According to Wil02, $\mathfrak{k}_{\xi}$ is a Lie ideal of $\mathfrak{g}_{\xi}$, therefore $K_{\xi}$ is a normal subgroup of $G_{\xi}$. Since $G_{\xi}$ is compact,

$$
K_{\xi}=\bigcup_{g \in G_{\xi}}\left(g\left(K_{\xi} \cap T\right) g^{-1}\right)
$$

is compact too. So c) is equivalent to (2).

Theorem 5.4. Suppose we are given a free Hamiltonian action of a compact group $G$ on a contact manifold $\left(M, \theta_{M}\right)$ and a non-zero element $\xi \in \mathfrak{g}^{*}$ satisfying a), b) and c) and suppose that the isotropy group $G_{\xi}$ is connected. Then Willett's reduced space $M_{\xi}^{W}$ (with a suitable choice of contact 1-form) is the prequantization of the reduced space $M_{[\xi]}$ obtained from the contact groupoid action of $S^{*} G$ with a suitable choice of reduction function $F$.

Proof. By Proposition 5.1, given a Hamiltonian action of $G$ on $\left(M, \theta_{M}\right)$, there is automatically a contact groupoid action of $S^{*} G$ on $\left(M, \theta_{M}\right)$. Since $G$ is compact, the function $f$ on the groupoid $S^{*} G$ is 1 (see Example 2.3). So we can choose as reduction function $F$ a constant function. We adopt the same notation as in Lemma 5.3. Then the reduction space

$$
M_{[\xi]}=J^{-1}([\xi]) / G_{\xi}=\phi^{-1}\left(\xi \cdot \mathbb{R}^{+}\right) / G_{\xi}
$$

is a symplectic manifold by Theorem 4.8, since $F$ is constant and $S\left(\mathfrak{g}^{*}\right)$ only has even-dimensional leaves.

Since $K_{\xi}$ is compact, the right action of $K_{\xi}$ on $G_{\xi}$ is proper. Notice that $G_{\xi}$ is connected and $K_{\xi}$ is a normal subgroup, so $G_{\xi} / K_{\xi}$ is a 1-dimensional compact connected group, therefore $S^{1}$. Let the quotient group $G_{\xi} / K_{\xi}$ act on $M_{\xi}^{W}$ by $[x] \cdot[g]=[x \cdot g]$. This action is free, and

$$
M_{\xi}^{W} /\left(G_{\xi} / K_{\xi}\right)=\phi^{-1}\left(\xi \cdot \mathbb{R}^{+}\right) / G_{\xi}=M_{[\xi]} .
$$

So $M_{\xi}^{W}$ is an $S^{1}$-principal bundle over $M_{[\xi]}$.

Now we claim that the $S^{1}$-principal bundle $M_{\xi}^{W}$ is furthermore a prequantization of $M_{[\xi]}$. From the construction in Section 4 , the symplectic form $\omega$ on $M_{[\xi]}$ is induced by the restriction of $-F^{-1} d\left(\|\phi\|^{-1} \theta_{M}\right)$ on $\phi^{-1}\left(\xi \cdot \mathbb{R}^{+}\right)$. We choose the contact 1-form $\theta_{W}$ on $M_{\xi}^{W}$ to be the one induced by the restriction of $-(F\|\phi\|)^{-1} \theta_{M}$ on $\phi^{-1}\left(\xi \cdot \mathbb{R}^{+}\right)$. Since Willett's reduction only depends on contact structures, we can choose any 
$G$-invariant contact form representing the same structure to do reduction. Here, by the equivariance of $\phi$, the new form $-(F\|\phi\|)^{-1} \theta_{M}$ is $G$-invariant and it is just a rescaling to $\theta_{M}$, so the level set of the new moment map is unchanged. Notice that the pullback of $\omega$ by $\pi: M_{\xi}^{W} \rightarrow M_{[\xi]}$ is exactly $d \theta_{W}$.

On $\phi^{-1}\left(\xi \cdot \mathbb{R}^{+}\right)$we have

$$
\theta_{M}\left(\xi_{M}\right)=\langle\phi, \xi\rangle=\|\phi\| \cdot\|\xi\|, \quad L_{\xi_{M}} \theta_{M}=0,
$$

where $\xi_{M}$ is the infinitesimal action generated by $\xi$. Using $d \theta_{M}\left(v_{M}, \cdot\right)=-d\langle\phi, v\rangle$ (see Proposition 3.1 in Wil02]) we see that $\phi_{*} \xi_{M}=0$, so $\xi_{M}$ is tangent to $\phi^{-1}\left(\xi \cdot \mathbb{R}^{+}\right)$. This and the fact that the function $\|\phi\|$ is invariant under the flow of $\xi_{M}$ imply that, on the quotient space $M_{\xi}^{W}$, the induced vector field $\left[-F \frac{\xi_{M}}{\|\xi\|}\right]$ is the Reeb vector field of $\theta_{W}$. However, in general, $\left[-F \frac{\xi_{M}}{\|\xi\|}\right]$ is not the generator of the $S^{1}$ action (cf. Example 5.10). Let

$$
t_{0}=\min _{t>0}\left\{\exp t \xi \in K_{\xi}\right\}
$$

Then the generator of the $G_{\xi} / K_{\xi}$ action is $t_{0}\left[\xi_{M}\right]$. Therefore, to finish the proof, we can just choose $F=-t_{0}\|\xi\|$, which only depends on $G$ and $\xi$ but not the action.

In fact, it is not hard to determine $t_{0}$, hence $F$. We might assume $\xi \in \mathfrak{t}^{*}$ and write $\xi$ as a multiple of an integer point,

$$
\xi=\frac{\|\xi\|}{\sqrt{n_{1}^{2}+\ldots+n_{k}^{2}}} \cdot\left(n_{1}, \ldots, n_{k}\right), \quad \operatorname{gcd}\left(n_{1}, \ldots, n_{k}\right)=1 .
$$

Let $T=\min _{t>0}\{\exp t \xi=1\}$ and let $S_{\xi}^{1}$ be the circle generated by $\xi$. Then $S_{\xi}^{1}$ intersects $K_{\xi}$ at finitely many points since they are both compact and the intersection of their Lie algebras is trivial. Then $t_{0}$ is

$$
t_{0}=\frac{T}{\sharp\left(S_{\xi}^{1} \cap K_{\xi}\right)} .
$$

It is not hard to see that $T$ is the smallest positive number for which $T \cdot \xi$ is an integer, hence $T=\sqrt{n_{1}^{2}+\ldots+n_{k}^{2}} /\|\xi\|$. And since $\xi \perp \mathfrak{k}_{\xi}$, by simple combinatorics, $S_{\xi}^{1}$ and $K_{\xi}$ intersect at $n_{1}^{2}+\ldots+n_{k}^{2}$ points. Therefore

$$
t_{0}=\left(\|\xi\| \sqrt{n_{1}^{2}+\ldots+n_{k}^{2}}\right)^{-1} .
$$

So $F=-\left(\sqrt{n_{1}^{2}+\ldots+n_{k}^{2}}\right)^{-1}$.

Remark 5.5. i) When $G$ is not a compact group it is harder to predict which statements hold in place of Lemma 5.3 and Theorem 5.4. Indeed, in that case one can have the non-compact subgroup $K_{\xi}$ acting properly on $\Phi^{-1}\left(\mathbb{R}^{+} \xi\right)$ (see the proof of Lemma 5.3), and furthermore the isotropy group of the groupoid at $\xi$ might no longer be $G_{\xi}$. (See Wil02, also see Example 6.5).

ii) If $G_{\xi}$ is not connected we can prove a statement analogous to Theorem 5.4 by suitably modifying Willett's reduction procedure (see Theorem 5.7 and Remark 5.9).

Remark 5.6. i) We also have a direct proof that the manifold $M_{[\xi]}$ of Theorem 5.4 is symplectic, as follows. Let a Lie group $G$ act freely on a contact manifold $\left(M, \theta_{M}\right)$ with moment map $\phi$, and assume that $\phi$ is transverse to $\xi \cdot \mathbb{R}^{+}$(here $\xi \in \mathfrak{g}^{*}$ is nonzero) and $G_{\xi}$ act properly on $\phi^{-1}\left(\xi \cdot \mathbb{R}^{+}\right)$. The lifted action to the symplectization $\left(M \times \mathbb{R},-d\left(e^{s} \theta_{M}\right)\right.$ is Hamiltonian with moment map $\tilde{\phi}=e^{s} \phi$. Since the actions 
of $G_{\xi}$ on $\tilde{\phi}^{-1}(\xi)$ and $\phi^{-1}\left(\xi \cdot \mathbb{R}^{+}\right)$are intertwined, by taking the Marsden-Weinstein reduction at $\xi$ we see that $\left(\phi^{-1}\left(\xi \cdot \mathbb{R}^{+}\right) / G_{\xi}, d\left(\theta_{M} /\|\phi\|\right)\right)$ is a symplectic manifold.

As a consequence of this, we obtain a quick proof of Willett's reduction result. Indeed, assume additionally that Willett's conditions a) and c) are satisfied, and consider

$$
\pi: \phi^{-1}\left(\xi \cdot \mathbb{R}^{+}\right) / K_{\xi} \rightarrow \phi^{-1}\left(\xi \cdot \mathbb{R}^{+}\right) / G_{\xi}
$$

The pullback of $d\left(\theta_{M} /\|\phi\|\right)$ via $\pi$ is non-degenerate on hyper-distributions transverse to $\operatorname{ker} \pi_{*}$, showing that $\theta_{M} /\|\phi\|$ is a contact 1 -form on $\phi^{-1}\left(\xi \cdot \mathbb{R}^{+}\right) / K_{\xi}$.

ii) In spite of the existence of a direct proof, the use of contact groupoids allows us to work in a general framework. It provides a unified treatment for both Willett's and Albert's (see Section 5.3) reduction and makes it possible to do reduction at a general point even in the case when $G$ is non-compact (see Example 6.5).

5.2. Application to the prequantization of coadjoint orbits. Kostant constructed prequantizations of coadjoint orbits for applications in representation theory, using tools from Lie theory [Kos70. Here, using Theorem [5.4, we can give a different description of Kostant's prequatization.

Let $G$ be a compact Lie group and let $M$ be $S^{*} G$ endowed with the contact form as in Example 2.3, which using left translation to identify $M$ with $S\left(\mathfrak{g}^{*}\right) \times G$ reads

$$
\theta_{M}(\delta \xi, \delta g)_{([\xi], g)}=\left\langle\frac{\xi}{\|\xi\|}, L_{g^{-1}} \delta g\right\rangle .
$$

Consider the right action of $G$ on $M$ obtained by taking the cotangent lift of the action of $G$ on itself by right multiplication. The action of $G$ and the infinitesimal action of $\mathfrak{g}$, using the above identification, read 12

$$
([\xi], g) h=\left(\left[A d_{h}^{*} \xi\right], g h\right), \quad v_{M}([\xi], g)=\left(\left[a d_{v}^{*} \xi\right], L_{g_{*}} v\right) .
$$

Since $\theta_{M}\left(\left[a d_{v}^{*} \xi\right], L_{g_{*}} v\right)_{([\xi], g)}=\|\xi\|^{-1}\langle\xi, v\rangle$, this action is Hamiltonian in the sense of (17) with moment map $\phi([\xi], g)=\|\xi\|^{-1} \xi$. According to Proposition [5.1, there is automatically a contact groupoid action of $S^{*} G$ on $M$, given by the moment map $J=[\phi]$ and $([\xi], g) \cdot([\eta], h)=\left(\left[A d_{h}^{*} \xi\right], g h\right)$. This action is actually the right action of $S^{*} G$ on itself by groupoid multiplication.

Before stating the theorem, let us recall Kostant's construction of prequantizations of coadjoint orbits Kos70, where the coadjoint orbits are endowed with the negative of the usual KKS (Kostant-Kirillov-Souriau, see [CdS01]) symplectic form. View $\mathbb{R}$ as a Lie algebra with the zero structure, then

$$
\left.2 \pi i \xi\right|_{\mathfrak{g}_{\xi}}: \mathfrak{g}_{\xi} \rightarrow \mathbb{R}
$$

is a Lie algebra homomorphism. Kostant Kos70] has proved that it can be integrated into a group homomorphism $\chi: G_{\xi} \rightarrow S^{1}$ iff the KKS symplectic form $\omega_{\xi}$ on the coadjoint orbit $O_{\xi}$ is integral. In this case, the prequantization bundle $L$ is simply

$$
G \times S^{1} / G_{\xi} \text {, by identifying }(g, s) \sim\left(g h, \chi(h)^{-1} s\right) .
$$

There is a natural 1-form $\left(\alpha_{\xi}, \frac{d s}{2 \pi}\right)$ on $G \times S^{1}$, where $\alpha_{\xi}$ is the left translation of $\xi$ on $G$ and $s$ is the coordinate on $S^{1}$. It turns out that it descends to a 1 -form $\theta_{L}$ on $L$, and that $\theta_{L}$ is exactly the connection 1 -form.

\footnotetext{
${ }^{12}$ Here $A d_{h}^{*}=L_{h}^{*} R_{h^{-1}}^{*}$ is a right action of $G$ on $\mathfrak{g}^{*}$ and so is $a d^{*}$. It preserves the bi-invariant metric, therefore it is a right action on $S\left(\mathfrak{g}^{*}\right)$, too.
} 
Theorem 5.7. Let $G$ be a compact Lie group, let $\xi \in \mathfrak{g}^{*}$, and assume that $G_{\xi}$ is connected. Then

i) the KKS symplectic form $\omega_{\xi}$ on the coadjoint orbit $O_{\xi}$ is integral iff $\xi$ is conjugate to an integer point $\left(d_{1}, \ldots, d_{k}\right)$;

ii) the contact reduction via groupoids $M_{[\xi]}$ is the coadjoint orbit $O_{\xi}$ through $\xi$ with the standard KKS symplectic form, with a suitable choice of the reduction function $F$;

iii) in the case of i), the quotient of the $S^{1}$-bundle $M_{\xi}^{W} \rightarrow O_{\xi}$ by $\mathbb{Z}_{n}$ is exactly Kostant's prequantization bundle $L$, where $n=\operatorname{gcd}\left(d_{1}, \ldots, d_{k}\right)$.

Remark 5.8. Statement i) above is well known and follows easily from the main construction of the proof.

Proof. Choose a bi-invariant metric on $\mathfrak{g}^{*}$ and choose a maximal torus as in Theorem 5.4. We adapt the notation used there, too. Then we might assume that $\xi \in \mathfrak{t}^{*}$ since all statements are dependent only on the conjugacy class of $\xi$.

The reduced space at $\xi$ of the contact groupoid action of $S^{*} G$ on $M$ is

$$
M_{[\xi]}=J^{-1}([\xi]) / G_{\xi}=G / G_{\xi}=O_{\xi} .
$$

Since the action of $S^{*} G$ on $M$ is the right action of $S^{*} G$ on itself, if we performed reduction using $F=1$, then by Example 4.7 we would obtain the Jacobi structure on $S^{*} G / S^{*} G=S\left(\mathfrak{g}^{*}\right)$ for which $\mathbf{s}:\left(S^{*} G, \theta_{M}\right) \rightarrow S\left(\mathfrak{g}^{*}\right)$ is a Jacobi map, i.e. the one whose Poissonisation is $\mathfrak{g}^{*}-0$ with the Lie-Poisson structure (see Example 2.3). Notice that the Jacobi structure of $S\left(\mathfrak{g}^{*}\right)$ is induced by the Poisson structure on its Poissonisation through the embedding as a unit sphere [CZ]. Let $\omega_{\xi}$ be the KKS form on $O_{\xi}$; then $\lambda \omega_{\xi}=\omega_{\lambda \xi}$. Therefore, by choosing $F=-\|\xi\|^{-1}$, we obtain that $M_{[\xi]}$ is symplectomorphic to $O_{\xi}$ endowed with the negative of the KKS form, which proves ii). With this choice for $F$ and the requirement that $d \theta_{W}$ is the pull-back of $\omega_{\xi}$, by a similar analysis as in Theorem 5.4. Willett's reduced contact form on $M_{\xi}^{W}$ is

$$
\theta_{W}=\frac{\|\xi\|}{\sqrt{n_{1}^{2}+\ldots+n_{k}^{2}}} \theta_{c}
$$

where $\theta_{c}$ is the connection 1-form of the $S^{1}$-principal bundle $M_{\xi}^{W} \rightarrow M_{[\xi]}$ obtained as in Theorem 5.4 .

If $\omega_{\xi}$ is integral, following Kostant, one can construct a prequantization bundle $L$ of $O_{\xi} \cong M_{[\xi]}$. Construct a morphism between the two $S^{1}$-principal bundles over $M_{[\xi]}$,

$$
\psi: M_{\xi}^{W}=G / K_{\xi} \rightarrow L=G \times S^{1} / G_{\xi}, \text { by }[g] \mapsto[(g, 1)] .
$$

It is well defined, since $\mathfrak{k}_{\xi}=\left.\operatorname{ker} 2 \pi i \xi\right|_{\mathfrak{g} \xi}$, which implies $K_{\xi} \subset \operatorname{ker} \chi$. Since $G_{\xi}$ acts on $S^{1}$ transitively via $\chi, \psi$ is surjective. The quotient group ker $\chi / K_{\xi}$ as a subgroup of $G_{\xi} / K_{\xi}=S^{1}$ is closed, therefore it is $\mathbb{Z}_{n}$ for some integer $n$. So ker $\chi=K_{\xi} \times \mathbb{Z}_{n}$, and $\psi$ is an $n$-covering map.

Moreover it is not hard to see that $\psi$ is $S^{1}$-equivariant (here we "identify" $G_{\xi} / K_{\xi}$ and $S^{1}$ via $\chi$ ), therefore $T \psi$ takes the infinitesimal generator of the first copy of $S^{1}\left(=G_{\xi} / K_{\xi}\right)$ to $n$ times the generator of the other $S^{1}$, and $\psi$ induces the identity map on the base $M_{[\xi]}$. Hence, we have

$$
\psi^{*} \theta_{L}=n \cdot \theta_{c} .
$$


Moreover, notice that $d \theta_{W}$ is the pullback of $\omega_{\xi}$ via projection $M_{\xi}^{W} \rightarrow M_{[\xi]}$, and that $\omega_{\xi}$ is the curvature form of $L$. So we have $d \theta_{W}=d \psi^{*} \theta_{L}$. Combining with (20) and (21), we have

$$
\theta_{W}=\psi^{*} \theta_{L} \text { and } n=\frac{\|\xi\|}{\sqrt{n_{1}^{2}+\ldots+n_{k}^{2}}} .
$$

Since $n$ is an integer, $\xi=n \cdot\left(n_{1}, \ldots, n_{k}\right)$ is an integer point and obviously $n=$ $\operatorname{gcd}\left(n \cdot n_{1}, \ldots, n \cdot n_{k}\right)$. Moreover $M_{\xi}^{W} / \mathbb{Z}_{n}$ is a $\left(G_{\xi} / K_{\xi}\right) / \mathbb{Z}_{n}=S^{1}$ principal bundle, and the morphism $\psi$ induces an isomorphism of principal bundles

$$
\tilde{\psi}: M_{\xi}^{W} / \mathbb{Z}_{n} \rightarrow L .
$$

The one form $\theta_{W}$ on $M_{\xi}^{W}$ descends to a one form on $M_{\xi}^{W} / \mathbb{Z}_{n}$, and the first equation in (22) shows that $\tilde{\psi}$ is an isomorphism between the $S^{1}$ principal bundle $M_{\xi}^{W} / \mathbb{Z}_{n}$ (equipped with this one form) and Kostant's prequantization bundle $L$. This proves iii) and one direction of i).

For the converse direction in i), suppose that $\xi=\left(d_{1}, \ldots, d_{k}\right)=n \cdot\left(n_{1}, \ldots, n_{k}\right)$ is an integer point. Then

$$
\frac{\|\xi\|}{\sqrt{n_{1}^{2}+\ldots+n_{k}^{2}}}=n=\operatorname{gcd}\left(d_{1}, \ldots, d_{k}\right) .
$$

By (20), $M_{\xi}^{W} / \mathbb{Z}_{n}$ is a prequantization of $M_{[\xi]}=O_{\xi}$, where the $\mathbb{Z}_{n}$ action is induced by the one of $S^{1}$. Therefore the symplectic form on $O_{\xi}$ is integral.

Remark 5.9. To remove the condition on the connectedness of $G_{\xi}$ we can replace the subgroup $K_{\xi}$ used in Willett's reduction by ker $\chi$. This is a good choice not only because Willett's contact reduction procedure still goes through with this replacement, but also because the analogs of Theorems 5.4 and 5.7 can be proven without the extra assumption of $G_{\xi}$ being connected.

Example 5.10 $(G=U(2))$. Let $G=U(2)$ and $\xi=\frac{1}{\sqrt{5}}\left(\begin{array}{ll}2 & 0 \\ 0 & 1\end{array}\right)$. Under a bi-invariant inner product $\left(v_{1}, v_{2}\right)=\operatorname{tr}\left(v_{1} v_{2}^{*}\right)$, one can identify $u^{*}(2)$ (Hermitian matrices) with $u(2)$ by $\xi \mapsto-i \xi$. Then $G_{\xi}=S^{1} \times S^{1}$ is the maximal torus embedded as diagonal matrices in $U(2)$. It is not hard to see that

$$
K_{\xi}=\left\{\left(\begin{array}{cc}
a & 0 \\
0 & a^{-2}
\end{array}\right):\|a\|=1\right\} .
$$

Now let $G$ act on $M=S^{*} G$ as described at the beginning of this section. Using the identification

$$
U(2) \cong S^{3} \times S^{1},\left(\begin{array}{cc}
a & \gamma \bar{b} \\
b & -\gamma \bar{a}
\end{array}\right) \mapsto\left(\left(\begin{array}{l}
a \\
b
\end{array}\right), \gamma\right)
$$

we easily compute that the groupoid reduction is $M_{[\xi]}=U(2) /\left(S^{1} \times S^{1}\right)=S^{2}$ and Willett's reduction is $M_{\xi}^{W}=U(2) / K_{\xi}=S^{3}$. If we choose the reduction function $F=-\sqrt{5}^{-1}$, then the symplectic form on $M_{[\xi]}$ is the area form, and $M_{\xi}^{W}=S^{3}$ is exactly the prequantization of $S^{2}$, which verifies Theorem [5.4.

Moreover, by taking different values of $\xi$, one recovers all $S^{1}$ principal bundles over $S^{2}$. Suppose $\xi=\frac{1}{\sqrt{m^{2}+n^{2}}}\left(\begin{array}{cc}m & 0 \\ 0 & n\end{array}\right)$, where $m \neq n$ are in $\mathbb{Z}$ and have greatest common divisor 1 . Then following exactly the same method above, one sees that $M_{\xi}^{W}$ is a lens space, namely the quotient $L(|m-n|, 1)$ of $S^{3}$ by the diagonal $\mathbb{Z}_{|m-n|}$ action. 
5.3. Relation to Albert's reduction. Given a Hamiltonian contact action of $G$ on $M$, one can also perform Albert's reduction [Alb89, which we now review. For any regular value $\xi \in \mathfrak{g}^{*}$ of $\phi$, let $\mathfrak{g}_{\xi}$ act on $Z:=\phi^{-1}(\xi)$ by

$$
\mathfrak{g}_{\xi} \rightarrow \chi(Z), \quad v \mapsto v_{M}-\langle\xi, v\rangle E,
$$

where $v \in \mathfrak{g}_{\xi}, v_{M}$ is the infinitesimal action of $\mathfrak{g}$ on $M$, and $E$ is the Reeb vector field on $M$. By Proposition 3.1 in Wil02 we have for all $v \in \mathfrak{g}$

$$
d\langle\phi, v\rangle=-i\left(v_{M}\right) d \theta_{M} .
$$

From this, it is easy to see that $E$ is tangent to the $\phi$-level sets. So the above action is a Lie algebra action. Assume the Reeb vector field is complete. Then on an open neighborhood of the identity in $G_{\xi}$, one has a new action ${ }_{n}$ on $Z$,

$$
x \cdot{ }_{n} \exp v=\varphi_{-\langle\xi, v\rangle}(x \cdot \exp v),
$$

where $\varphi_{t}$ is the flow of $E$ and $x \cdot \exp v$ is the old action of $G$ on $M$. For simplicity, let us assume this action is free and proper and $G_{\xi}$ is connected. Then one can extend the new action to the whole of $G_{\xi}$ by multiplication in $G_{\xi}$ (BtD95]). Albert's reduction is defined as 13

$$
M_{\xi}^{A}:=Z / G_{\xi},
$$

with the contact structure inherited from $M$.

Now we show the relation between Albert's reduced spaces and ours. First of all, with the same set-up as for Albert's reduction and using the notation of Example 2.4, we have

Proposition 5.11. The action of $T^{*} G \times \mathbb{R}$ on $\left(M, \theta_{M}\right)$ given by

$$
m \cdot(\xi, g, r)=\varphi_{r}(m \cdot g)
$$

is a contact groupoid action with moment map $\phi$, where $\varphi_{r}$ is the time-r flow of the Reeb vector field $E$ on $M$. Here we identify $T^{*} G \times \mathbb{R}$ and $\mathfrak{g}^{*} \rtimes G \times \mathbb{R}$ by right translation.

Proof. Since the $G$ action preserves $E$ (because it preserves $\theta_{M}$ ), we have $\varphi_{r}(\mathrm{~m} \cdot \mathrm{g})=$ $\varphi_{r}(m) \cdot g$. So,

$$
\phi(m \cdot(\xi, g, r))=\phi\left(\varphi_{r}(m) \cdot g\right)=\phi\left(\varphi_{r}(m)\right) \cdot g=\phi(m) \cdot g=\mathbf{s}(\xi, g, r) .
$$

It is not hard to verify that the other conditions in the definition of groupoid action are satisfied. Furthermore, using the fact that $\theta_{M}$ is preserved by both $\varphi_{r}$ and the $G$ action, it is easy to check (4). Therefore the given action is a contact groupoid action.

Notice that the Lie algebra action (24) sits inside the bigger Lie algebra action

$$
\mathfrak{g}_{\xi} \times \mathbb{R} \rightarrow \chi(Z), \quad(v, r) \mapsto v_{M}+r E
$$

via the Lie algebra morphism $i: \mathfrak{g}_{\xi} \hookrightarrow \mathfrak{g}_{\xi} \times \mathbb{R}$ defined by $v \mapsto(v,-\langle\xi, v\rangle)$.

The isotropy group of $T^{*} G \times \mathbb{R}$ at $\xi$ is $G_{\xi} \times \mathbb{R}$, and its action corresponds exactly to the infinitesimal action above. If this action is free, then the reduction via contact groupoids

$$
M_{\xi}=Z /\left(G_{\xi} \times \mathbb{R}\right)
$$

\footnotetext{
${ }^{13}$ It coincides with $Z / \tilde{G}_{\xi}$, where $\tilde{G}_{\xi}$ is the simply connected group covering $G_{\xi}$ acting on $Z$ by the lift of the action $\cdot n$.
} 
is a symplectic manifold. Let $\tilde{G}_{\xi}$ be the simply connected Lie group covering $G_{\xi}$. Then, the above embedding $i$ gives a Lie group morphism (not necessarily injective any more)

$$
\bar{i}: \tilde{G}_{\xi} \rightarrow G_{\xi} \times \mathbb{R} .
$$

Then $H:=\mathbb{R} / \bar{i}\left(\tilde{G}_{\xi}\right) \cap \mathbb{R}$ acts on $Z / G_{\xi}$ freely. The quotient $H$ can be very singular if $\bar{i}\left(\tilde{G}_{\xi}\right) \cap \mathbb{R}$ is not discrete. If it is discrete, then $H$ is either $\mathbb{R}$ or $S^{1}$. In this case, we will have a $H$-principal bundle $\pi: M_{\xi}^{A} \rightarrow M_{\xi}$.

The contact 1-form $\theta_{\xi}$ on $M_{\xi}^{A}$ and the symplectic 2-form $\omega_{\xi}$ on $M_{\xi}$ are induced by $\theta_{M}$ and $d \theta_{M}$ on $Z$ with $F=-1$. Hence $\pi^{*} \omega_{\xi}=d \theta_{\xi}$. The Reeb vector field on $M$ descends to the Reeb vector field on $M_{\xi}^{A}$. Since $\mathbb{R}$ acts by Reeb flows, the generator of $H$ is a multiple of the Reeb vector field on $M_{\xi}^{A}$. Therefore if $H \cong S^{1}$, similar to the discussion of Willett's reduction, one can rescale the reduction function $F$ suitably to make $M_{\xi}^{A}$ a prequantization of $M_{\xi}$. If $H \cong \mathbb{R}$, then $M_{\xi}^{A}$, being a $\mathbb{R}$-principal bundle over $M_{\xi}$, is simply $M_{\xi} \times \mathbb{R}$. Summarizing we obtain:

Theorem 5.12. Let $M_{\xi}$ be the contact groupoid reduction via $T^{*} G \times \mathbb{R}$ at the point $\xi$, let $M_{\xi}^{A}$ be the Albert reduction space at $\xi$ and let $H$ be the group defined above. If the groupoid action of $T^{*} G \times \mathbb{R}$ is free and $H$ is either $\mathbb{R}$ or $S^{1}$, then

(1) $M_{\xi}^{A}$ is a prequantization of $M_{\xi}$ if $H=S^{1}$;

(2) $M_{\xi}^{A}=M_{\xi} \times \mathbb{R}$ if $H=\mathbb{R}$.

\section{EXAMPLES}

In this section we will exhibit some examples of contact groupoid reduction using Theorem 4.1. We start by describing the general strategy we use to apply the above theorem.

(1) Given a contact manifold $\left(M, \theta_{M}\right)$ and an integrable Jacobi manifold $\Gamma_{0}$, choose a complete Jacobi map $J: M \rightarrow \Gamma_{0}$.

(2) Let $\Gamma$ be the t-simply connected contact groupoid of $\Gamma_{0}$. For any choice of $x$ lying in a contact leaf of $\Gamma_{0}$, restricting the Lie algebroid action $J^{*}\left(\left.\operatorname{ker} \mathbf{t}_{*}\right|_{\Gamma_{0}}\right) \rightarrow T M, X_{\mathbf{s}^{*} u} \mapsto X_{J^{*} u}$, obtain the Lie algebra action of $T_{x} \Gamma_{x}$ on $J^{-1}(x)$.

(3) Integrating determine the Lie group action of $\Gamma_{x}$ on $J^{-1}(x)$.

(4) Choose an $f$-multiplicative function $F$ on $J^{-1}(x)$ (or an open subset thereof).

(5) If the quotient of $J^{-1}(x)$ (or an open subset thereof) by $\Gamma_{x}$ is a manifold, then it is a contact manifold equipped with the one form induced by $-F^{-1} \theta_{M}$.

We wish to explain in detail how to obtain the Lie algebra action of $T_{x} \Gamma_{x}$ on $J^{-1}(x)$ in (2). By Theorem 3.8 the map $J$ in (1) induces a (contact) groupoid action on $\Gamma$ on $M$. From the construction in Theorem 3.8 it is clear that the induced Lie algebroid action 14 is $J^{*}\left(\left.\operatorname{ker} \mathbf{t}_{*}\right|_{\Gamma_{0}}\right) \rightarrow T M,\left(X_{\mathbf{s}^{*} u}(J(m)), m\right) \mapsto X_{J^{*} u}(m)$. Here $u$ is

\footnotetext{
${ }^{14}$ Given any Lie groupoid $\Gamma \rightrightarrows \Gamma_{0}$ the associated Lie algebroid is ker $\left.\mathbf{t}_{*}\right|_{\Gamma_{0}} \rightarrow \Gamma_{0}$, and any groupoid action of $\Gamma$ on a map $J: M \rightarrow \Gamma_{0}$ induces a Lie algebroid action of ker $\left.\mathbf{t}_{*}\right|_{\Gamma_{0}}$ by differentiating curves $m \cdot g(t)$, where $m \in M$ and $g(t)$ is a curve in $\mathbf{t}^{-1}(J(m))$ passing through $J(m)$ at time zero (see [CdSW99]). Above $J^{*}\left(\left.\operatorname{ker} \mathbf{t}_{*}\right|_{\Gamma_{0}}\right)$ denotes the vector bundle on $M$ obtained by pullback via $J$.
} 
a smooth function on $\Gamma_{0}$. Restricting to $T_{x} \Gamma_{x}=\operatorname{ker}\left(\mathbf{t}_{*}\right)_{x} \cap \operatorname{ker}\left(\mathbf{s}_{*}\right)_{x}$ we obtain a map $J^{*}\left(T_{x} \Gamma_{x}\right) \rightarrow T J^{-1}(x)$, i.e. a map

$$
T_{x} \Gamma_{x} \rightarrow \chi\left(J^{-1}(x)\right),\left.X_{\mathbf{s}^{*} u}(x) \mapsto X_{J^{*} u}\right|_{J^{-1}(x)} .
$$

Being obtained by restriction, this will be the infinitesimal action associated to the Lie group action of $\Gamma_{x}$ on $J^{-1}(x)$. Therefore, to obtain explicitly the $\Gamma_{x}$-action, all we have to do is to integrate the above Lie algebra action. If the group action of $\Gamma_{x}$ on $J^{-1}(x)$ is free and proper, then a similar proof as in Proposition-Definition 3.10 ensures the existence on a function $F$ as above on $J^{-1}(x)$ and the quotient $J^{-1}(x) / \Gamma_{x}$ will be smooth.

Remark 6.1. In the first three examples below we will have $\Gamma_{0}=(\mathbb{R}, d t)$. Let us explicitly describe its t-simply connected contact groupoid $\Gamma$ (see KSB93 for the case where $\Gamma_{0}$ is a general contact manifold). We have

$$
\left(\Gamma=\mathbb{R} \times \mathbb{R} \times \mathbb{R}, \theta_{\Gamma}=-e^{-s} d p+d q, f=e^{-s}\right),
$$

where we use coordinates $(p, q, s)$ on $\Gamma$. Therefore the Reeb vector field is $E_{\Gamma}=\frac{\partial}{\partial q}$ and $\Lambda_{\Gamma}=\frac{\partial}{\partial s} \wedge\left(e^{s} \frac{\partial}{\partial p}+\frac{\partial}{\partial q}\right)$. The groupoid structure is given by $\mathbf{t}(p, q, s)=p$, $\mathbf{s}(p, q, s)=q$ and $(p, q, s)(\tilde{p}, \tilde{q}, \tilde{s})=(p, \tilde{q}, s+\tilde{s})$ when $q=\tilde{p}$, so the isotropy groups are given by $\Gamma_{x}=\{x\} \times\{x\} \times \mathbb{R}$.

Example 6.2. On $M=\mathbb{R}^{2 n+1}$ we choose standard coordinates $\left(x_{1}, \ldots, x_{n}, y_{1}, \ldots\right.$, $\left.y_{n}, z\right)$, concisely denoted by $\left(x_{i}, y_{i}, z\right)$. Consider

$$
J:\left(\mathbb{R}^{2 n+1}, \sum_{i=1}^{n} x_{i} d y_{i}-y_{i} d x_{i}+d z\right) \rightarrow(\mathbb{R}, d t),\left(x_{i}, y_{i}, z\right) \mapsto z .
$$

Notice that this is indeed a Jacobi map since $E_{M}=\frac{\partial}{\partial z}$ and

$$
\Lambda_{M}=\frac{1}{2} \sum\left(\frac{\partial}{\partial x_{i}}+y_{i} \frac{\partial}{\partial z}\right) \wedge\left(\frac{\partial}{\partial y_{i}}-x_{i} \frac{\partial}{\partial z}\right) .
$$

Therefore the Lie algebroid action (or rather the induced map from sections of ker $\left.\mathbf{t}_{*}\right|_{\Gamma_{0}}$ to vector fields on $M$ ) is given by

$$
X_{\mathbf{s}^{*} u}=u \cdot \frac{\partial}{\partial q}-u^{\prime} \cdot \frac{\partial}{\partial s} \mapsto X_{J^{*} u}=u(z) \frac{\partial}{\partial z}+\frac{1}{2} u^{\prime}(z) \sum x_{i} \frac{\partial}{\partial x_{i}}+y_{i} \frac{\partial}{\partial y_{i}} .
$$

Notice that the formula for $X_{J^{*} u}$ implies that $J$ is a complete map. Indeed, if $u$ is a compactly supported function on $\Gamma_{0}$, then we have $\left|X_{J^{*} u}(m)\right| \leq C \cdot r$ at all $m \in \mathbb{R}^{2 n+1}$, where $r$ is the distance of $m$ from the origin and $C$ some constant. Therefore at time $t$ the integral curve of $X_{J^{*}} u$ passing through $m_{0}$ will have distance at most $\left|m_{0}\right| e^{C r t}$ from the origin, and hence it will be defined for all time.

Choosing $\bar{t}=0 \in \Gamma_{0}$ we obtain the Lie algebra action $15 T_{\bar{t}} \Gamma_{\bar{t}}=\mathbb{R} \rightarrow J^{-1}(0)=$ $\mathbb{R}^{2 n}$ with infinitesimal generator $-\frac{1}{2} \sum\left(x_{i} \frac{\partial}{\partial x_{i}}+y_{i} \frac{\partial}{\partial y_{i}}\right)$, so the Lie group action of $\Gamma_{\bar{t}}$ on $J^{-1}(0)$ is given by $\left(x_{i}, y_{i}\right) \cdot s=\left(e^{-\frac{1}{2} s} x_{i}, e^{-\frac{1}{2} s} y_{i}\right)$. Since $f=e^{-s}$ we can choose $F=\sum x_{i}^{2}+y_{i}^{2}$. Notice that the action is not free at the origin (not even locally free). Using the fact that each $\Gamma_{x}$-orbit intersects the unit sphere exactly once, we see that the quotient of $\left(\mathbb{R}^{2 n}-\{0\},-\frac{\sum x_{i} d y_{i}-y_{i} d x_{i}}{\sum x_{i}^{2}+y_{i}^{2}}\right)$ by the $\mathbb{R}$-action is

$$
\left(S^{2 n-1},-\left(\sum x_{i} d y_{i}-y_{i} d x_{i}\right)\right)
$$

\footnotetext{
${ }^{15}$ As usual here $\Gamma_{\bar{t}}$ denotes the isotropy group of $\Gamma$ at $\bar{t}$.
} 
i.e. up to sign the standard contact form for the unit sphere in $\mathbb{R}^{2 n}$.

Remark 6.3. In the above example the groupoid action of $\Gamma$ on $M$ is given by

$$
\left(x_{i}, y_{i}, z\right) \cdot(p, q, s)=\left(e^{-\frac{1}{2} s} x_{i}, e^{-\frac{1}{2} s} y_{i}, q\right)
$$

whenever $z=p$, and one can explicitly check that formula (4) in the definition of contact groupoid action holds. Also notice that $J$ is a submersion everywhere, however at $m \in\{0\} \times \mathbb{R} \subset \mathbb{R}^{2 n+1}$ the tangent space to the $J$-fiber and ker $\theta_{M}$ coincide, so that - as stated in Lemma 3.5 at such points $m$ the groupoid action is not locally free.

Example 6.4 (Cosphere bundle). Let $N$ be any manifold, endowed with a Riemannian metric, and let $M=T^{*} N \times \mathbb{R}$. Consider

$$
J:\left(T^{*} N \times \mathbb{R}, \alpha+d z\right) \rightarrow(\mathbb{R}, d t),(\xi, z) \mapsto z .
$$

Here $\alpha$ is the canonical one-form on $T^{*} N$, i.e. with respect to local coordinates $\left\{x_{i}\right\}$ on $N$ and $\left\{y_{i}\right\}$, which are the coordinates with respect to the dual basis of $\left\{\frac{\partial}{\partial x_{i}}\right\}$ (giving coordinates $\left\{x_{i}, y_{i}\right\}$ on $T^{*} N$ ), it is just $\sum y_{i} d x_{i}$. In local coordinates we have $E_{M}=\frac{\partial}{\partial z}$ and $\Lambda_{M}=\sum \frac{\partial}{\partial y_{i}} \wedge\left(\frac{\partial}{\partial x_{i}}-y_{i} \frac{\partial}{\partial z}\right)$. Therefore the Lie algebroid action is given by

$$
X_{\mathbf{s}^{*} u}=u \frac{\partial}{\partial q}-u^{\prime} \frac{\partial}{\partial s} \mapsto X_{J^{*} u}=u(z) \frac{\partial}{\partial z}+u^{\prime}(z) \sum y_{i} \frac{\partial}{\partial y_{i}} .
$$

The above expression for $\left\|X_{J^{*} u}\right\|$ ensures that $J$ is a complete map.

Choosing $\bar{t}=0 \in \Gamma_{0}$ we obtain as infinitesimal generator of the Lie algebra action the radial vector field $-\sum y_{i} \frac{\partial}{\partial y_{i}}$. The Lie group action of $\Gamma_{\bar{t}}$ on $J^{-1}(0)$ is given in local coordinates by $\left(x_{i}, y_{i}\right) \cdot s=\left(x_{i}, y_{i} e^{-s}\right)$, i.e. by $\xi \cdot s=\xi \cdot e^{-s}$, where $\xi \in T_{p}^{*} N$. We choose $F=\|\xi\|$ and note that the action is free on $T^{*} N-\{0\}$. Each $\Gamma_{0}$-orbit there intersects the unit cosphere bundle $T_{1}^{*} N$ (the set of covectors of length one) exactly once. Since by Theorem 4.1 the one-form $-\frac{\alpha}{\|\varepsilon\|}$ on $T^{*} N-\{0\}$ is basic w.r.t. the natural projection, we conclude that $T_{1}^{*} N \cong\left(T^{*} N-\{0\}\right) / \Gamma_{\bar{t}}$ endowed with the one-form $-\left.\alpha\right|_{T_{1}^{*} N}$ is a contact manifold.

Now we present an example where Willett's reduction fails but contact groupoid reduction works.

Example 6.5 (Non-compact group $G=S L(2, \mathbb{R})$ ). Let $G$ be a Lie group and let $G$ act on $M=\left(T^{*} G-G\right) \times \mathbb{R}$ from the right by $(\xi, g, t) h=\left(A d_{h}^{*} \xi, g h, t\right)$. Here we identify $T^{*} G$ with $\mathfrak{g}^{*} \times G$ by left translation. By a calculation similar to the one at the beginning of subsection 5.2, we can see that this is a Hamiltonian action with moment map $\phi(\xi, g, t)=\xi$. By Claim 5.1 the cosphere bundle $S^{*} G$ as a contact groupoid automatically acts on $M$. Let $G=S L(2, \mathbb{R})$. Then we are actually revisiting Example 3.7 in Wil02, except that we adapt everything to right actions. In Wil02 it is shown that Willett's reduction at the point $\xi=\left(\begin{array}{ll}0 & 1 \\ 0 & 0\end{array}\right)$ has four dimensions, therefore it is not a contact manifold.

However, the reduction by contact groupoids is a contact manifold. Using the standard Killing form on $S L(2, \mathbb{R})$, that is $\langle X, Y\rangle=\operatorname{tr}(X \cdot Y)$, we identify $s l^{*}(2, \mathbb{R})$ and $\operatorname{sl}(2, \mathbb{R})$. Then the isotropy group $\Gamma_{[\xi]}$ of the groupoid is

$$
\Gamma_{[\xi]}=\left\{\left(\begin{array}{cc}
\alpha & \gamma \\
0 & \alpha^{-1}
\end{array}\right): \alpha \in \mathbb{R}-0, \gamma \in \mathbb{R}\right\},
$$


which has one more dimension than the stabilizer group $G_{\xi}$. Let $B$ be the Borel subgroup of $S L(2, \mathbb{R})$ embedded as upper triangular matrices. Then $B$ is a normal subgroup of $\Gamma_{[\xi]}$ and $\Gamma_{[\xi]}=\mathbb{R}^{+} \times \mathbb{Z}_{2} \times B$.

We want to quotient out

$$
J^{-1}([\xi])=\left\{(\lambda \xi, g, t) \mid \lambda \in \mathbb{R}^{+}, g \in S L(2, \mathbb{R}), t \in \mathbb{R}\right\}
$$

by $\Gamma_{\xi}$. Note that $S L(2, \mathbb{R})$ acts on $\mathbb{R}^{2}-0$ transitively with stabilizer $B$ at the point $(1,0)$. So $S L(2, \mathbb{R}) / B=\mathbb{R}^{2}-0$. Therefore, by a more careful examination of the quotient space $J^{-1}([\xi]) / \Gamma_{\xi}$,

$$
M_{[\xi]}=\left(\left(\mathbb{R}^{2}-0\right) / \mathbb{Z}_{2}\right) \times \mathbb{R}=\left(\mathbb{R}^{2}-0\right) \times \mathbb{R} .
$$

It is not surprising at all that we get a contact manifold by the groupoid reduction at $[\xi]=\left[\left(\begin{array}{ll}0 & 1 \\ 0 & 0\end{array}\right)\right]$, since $[\xi]$ lies in a contact leaf of $S\left(s l^{*}(2, \mathbb{R})\right)$. Indeed, identify $s l^{*}(2, \mathbb{R})$ with $\mathbb{R}^{3}$ by a series of new coordinate functions:

$$
\begin{aligned}
\mu_{1} & =\frac{1}{2}(X+Y), \\
\mu_{2} & =\frac{1}{2} H, \\
\mu_{3} & =\frac{1}{2}(X-Y),
\end{aligned}
$$

where $X=\left(\begin{array}{ll}0 & 1 \\ 0 & 0\end{array}\right), Y=\left(\begin{array}{ll}0 & 0 \\ 1 & 0\end{array}\right)$ and $H=\left(\begin{array}{cc}1 & 0 \\ 0 & -1\end{array}\right)$ are the standard generators of $s l(2, \mathbb{R})$. Then the symplectic leaves of $s l^{*}(2, \mathbb{R})$ sitting inside $\mathbb{R}^{3}$ are level surfaces of the Casimir function $\mu_{1}^{2}+\mu_{2}^{2}-\mu_{3}^{2}$. That is, they are hyperbolas of two sheets and one sheet as well as symplectic cones. Then $\xi=(1,0,1)$ lies inside a symplectic cone, which induces a contact leaf on $S\left(s l^{*}(2, \mathbb{R})\right)$ because the radial vector of the symplectic cone gives exactly the infinitesimal action of $\mathbb{R}^{+}$, by which we quotient out to get the Jacobi structure on $S\left(s l^{*}(2, \mathbb{R})\right)$.

Remark 6.6. It turns out that every point $\xi$ of a nilpotent adjoint orbit of a semisimple Lie algebra can give rise to a contact manifold as above. This is under further investigation.

Example 6.7 (Variation with non-compact group $G=S L(3, \mathbb{R})$ ). In Example 6.5. we saw that the action of a group $G$ on the contact manifold ( $M=$ $\left.\left(T^{*} G-G\right) \times \mathbb{R}, \theta_{c}+d t\right)$ from the right by $(\xi, g, t) h=\left(A d_{h}^{*} \xi, g h, t\right)$ is a Hamiltonian action, with moment map $\phi(\xi, g, t)=\xi$. Now we choose $G=S L(3, \mathbb{R})$, and we obtain a Hamiltonian action of $S L(2, \mathbb{R})$ on $M$ by restricting the above action to $S L(2, \mathbb{R}) \subset S L(3, \mathbb{R})$ (the embedding is given by $H \mapsto\left(\begin{array}{cc}H & 0 \\ 0 & 1\end{array}\right)$ ). Then, using the Killing form $\langle X, Y\rangle=\operatorname{tr}(X Y)$ to identify a Lie algebra with its dual and identifying $M$ with $\left(s l^{*}(3, \mathbb{R})-0\right) \times S L(3, \mathbb{R}) \times \mathbb{R}$ by left translations, the moment map of the Hamiltonian action reads

$$
\phi:\left(s l^{*}(3, \mathbb{R})-0\right) \times S L(3, \mathbb{R}) \times \mathbb{R} \rightarrow s l^{*}(2, \mathbb{R}),\left(\left(\begin{array}{ll}
A & b \\
c & d
\end{array}\right), g, t\right) \mapsto A+\frac{d}{2}\left(\begin{array}{ll}
1 & 0 \\
0 & 1
\end{array}\right) .
$$

By Proposition 5.1 we have an induced action of the contact groupoid of the sphere $S\left(s l^{*}(2, \mathbb{R})\right)$ on $M$, with moment map $J=[\phi]$. Now we will perform contact groupoid reduction at the point $[\xi]=\left[\left(\begin{array}{ll}0 & 1 \\ 0 & 0\end{array}\right)\right]$, which lies in a contact leaf of 
$S\left(s l^{*}(2, \mathbb{R})\right)$. The reduced space is the quotient of

$$
\begin{gathered}
J^{-1}([\xi])=\left\{\left(\begin{array}{ccc}
-\frac{d}{2} & \lambda & b_{1} \\
0 & -\frac{d}{2} & b_{2} \\
c_{1} & c_{2} & d
\end{array}\right), g, t\right) \quad: \quad \lambda \in \mathbb{R}_{+}, b_{1}, b_{2}, c_{1}, c_{2}, d \in \mathbb{R} ; \\
\\
g \in S L(3, \mathbb{R}) ; t \in \mathbb{R}\}
\end{gathered}
$$

by $\Gamma_{[\xi]}=\left\{\left(\begin{array}{cc}\alpha & \gamma \\ 0 & \alpha^{-1}\end{array}\right): \alpha \in \mathbb{R}-0, \gamma \in \mathbb{R}\right\}$, which is the isotropy group at $[\xi]$ of the groupoid. Explicitly, the action is given by

$$
\left(\left(\begin{array}{ll}
A & b \\
c & d
\end{array}\right), g, t\right) \cdot H=\left(\left(\begin{array}{cc}
H^{-1} A H & H^{-1} b \\
c H & d
\end{array}\right), g \cdot\left(\begin{array}{cc}
H & 0 \\
0 & 1
\end{array}\right), t\right),
$$

where $\left(\left(\begin{array}{ll}A & b \\ c & d\end{array}\right), g, t\right)$ and $H \in \Gamma_{\xi}$. As in Example 6.5 we will reduce first by the Borel subgroup $\left\{\left(\begin{array}{ll}1 & \gamma \\ 0 & 1\end{array}\right): \gamma \in \mathbb{R}\right\}$ and then by $\left\{\left(\begin{array}{cc}\alpha & 0 \\ 0 & \alpha^{-1}\end{array}\right): \alpha \in \mathbb{R}-0\right\}$. To simplify the computation identify $S L(3, \mathbb{R})$ with $U \times \mathbb{R}^{2}$ by identifying $\left(\begin{array}{ccc}1 & 1 & 1 \\ \mathbf{v} & \mathbf{w} & \mathbf{z} \\ 1 & 1 & \mid\end{array}\right)$ with $(\mathbf{v}, \mathbf{z}, \nu, \eta)$, where $\mathbf{w}=\frac{\mathbf{v} \times \mathbf{z}}{|\mathbf{v} \times \mathbf{z}|^{2}}+\nu \mathbf{v}+\eta \mathbf{z}$. Here

$$
U=\left\{\text { pairs of linearly independent vectors in } \mathbb{R}^{3}\right\}=\left(\mathbb{R}^{3}-0\right) \times\left(\mathbb{R}^{3}-\mathbb{R}\right) .
$$

The resulting quotient is

$$
\left(\mathbb{R}^{3}-\mathbb{R}\right) \times \mathbb{R}^{3} \times\left(S^{2} \times \mathbb{R}^{5}\right) / \mathbb{Z}_{2} .
$$

Since $\left(S^{2} \times \mathbb{R}^{5}\right) / \mathbb{Z}_{2}$ embeds in $\left(\mathbb{R}^{8}-0\right) / \mathbb{Z}_{2}$ (which is an $\mathbb{R}^{+}$bundle over $\mathbb{R P}^{7}$ ) as a section of the $\mathbb{R}^{+}$-bundle defined over $\left\{\left[\left(x_{1}, \cdots, x_{8}\right)\right]: x_{1}, x_{2}, x_{3} \neq 0\right\} \subset \mathbb{R P}^{7}$, our quotient can be re-written as

$$
S^{1} \times \mathbb{R}^{5} \times\left(\mathbb{R P}^{7}-\mathbb{R P}^{4}\right)
$$

Remark 6.8. The examples exhibited here are all well-known examples of contact manifolds, as one can see using, for example, Theorem 3.6 in Bla02].

\section{APPENDIX I-INVARIANCE OF CONTACT STRUCTURES}

To prove the invariance of the contact structure on the reduced space, we present in this appendix a "form-free" version (Appendix I, Theorem 1.4) of our main results (Theorem 4.1 and Theorem 4.4). As stated in Section 2, we assume that all contact structures involved in this paper are co-oriented, but the next two definitions make sense even without this assumption.

First, let us recall the definition of conformal contact groupoid 16 from Daz97.

Definition 1.1. A Lie groupoid $\Gamma$ together with a contact structure (i.e. a contact hyperplane distribution) $\mathcal{H}_{\Gamma}$ is called a conformal contact groupoid if

i) $(X, Y) \in \mathcal{H}_{\Gamma} \times \mathcal{H}_{\Gamma} \Rightarrow X \cdot Y \in \mathcal{H}_{\Gamma}$, whenever $X \cdot Y$ is defined;

ii) the inversion $i: \Gamma \rightarrow \Gamma$ leaves $\mathcal{H}_{\Gamma}$ invariant.

Definition 1.2. Let $\left(\Gamma, \mathcal{H}_{\Gamma}\right)$ be a conformal contact groupoidand let $M$ be a manifold with contact structure $\mathcal{H}_{M}$. A (right) groupoid action $\Phi$ of $\Gamma$ on $M$ is a conformal contact groupoidaction if

i) $(Y, V) \in \mathcal{H}_{M} \times \mathcal{H}_{\Gamma} \Rightarrow \Phi_{*}(Y, V) \in \mathcal{H}_{M}$,

ii) $Y \in \mathcal{H}_{M}, \Phi_{*}(Y, V) \in \mathcal{H}_{M} \Rightarrow V \in \mathcal{H}_{\Gamma}$, whenever $\Phi_{*}(Y, V)$ is defined.

\footnotetext{
${ }^{16} \mathrm{It}$ is known under various names in the literature. Here we use the same name as in [CZ].
} 
Remark 1.3. Condition ii) implies that for the Reeb vector field of any contact one-form $\theta_{\Gamma}$ with kernel $\mathcal{H}_{\Gamma}$,

$$
0 \cdot E_{\Gamma} \notin \mathcal{H}_{M} .
$$

In fact, it is not hard to deduce from the proof of Lemma 1.7 that (25) is equivalent to condition (ii).

Theorem 1.4. Let $\left(M, \mathcal{H}_{M}\right)$ be a manifold with a contact structure and let $\Phi$ be a conformal contact groupoidaction of $\left(\Gamma, \mathcal{H}_{\Gamma}\right)$ on $\left(M, \mathcal{H}_{M}\right)$. Then the point-wise reduced spaces $J^{-1}(x) / \Gamma_{x}$ naturally inherit a contact or conformal l.c.s. structure, and they are exactly the leaves of the global reduced space $M / \Gamma$ endowed with the conformal Jacobi structure as in Theorem 4.4 .

We start with a lemma involving only groupoids and not actions:

Lemma 1.5. Let $\left(\Gamma, \mathcal{H}_{\Gamma}\right)$ be a conformal contact groupoid. Then

i) there is a multiplicative function $f$ on $\Gamma$ and a contact form $\theta_{\Gamma}$ with kernel $\mathcal{H}_{\Gamma}$ such that the triple $\left(\Gamma, f, \theta_{\Gamma}\right)$ is a contact groupoid.

ii) $\left(\Gamma, \hat{f}, \hat{\theta}_{\Gamma}\right)$ is another such triple if and only if there is a non-vanishing function $u$ on $\Gamma_{0}$ such that $\hat{f}=f \frac{\mathbf{s}^{*} u}{\mathbf{t}^{*} u}$ and $\hat{\theta}_{\Gamma}=\mathbf{s}^{*}(u) \theta_{\Gamma}$.

Proof. i) is the remark following Proposition 4.1 in Daz97. We will indicate the proof of ii). Given a contact groupoid $\left(\Gamma, f, \theta_{\Gamma}\right)$, using the fact that $\frac{\mathbf{s}^{*} u}{\mathbf{t}^{*} u}$ is multiplicative, it is not hard to verify equation (3) for the triple $\left(\Gamma, f \frac{\mathbf{s}^{*} u}{\mathbf{t}^{*} u}, \mathbf{s}^{*} u \theta_{\Gamma}\right)$, so that it is again a contact groupoid. Conversely suppose that $\left(\Gamma, \hat{f}, \hat{\theta}_{\Gamma}\right)$ is a contact groupoid. Then there exist a multiplicative function $\phi$ on $\Gamma$ and a non-vanishing function $\tau$ on $\Gamma$ such that $\hat{f}=\phi f$ and $\hat{\theta}_{\Gamma}=\tau \theta_{\Gamma}$. Therefore the multiplication $\omega$ satisfies

$$
\omega^{*}\left(\tau \theta_{\Gamma}\right)=p r_{2}^{*}(\phi f) \cdot p r_{1}^{*}\left(\tau \theta_{\Gamma}\right)+p r_{2}^{*}\left(\tau \theta_{\Gamma}\right) .
$$

Evaluating this at $(g, h) \in \Gamma_{\mathbf{s}} \times_{\mathbf{t}} \Gamma$ and using Lemma 4.1 in Daz97, we obtain $\tau(g h)=\tau(h)=\phi(h) \tau(g)$. The first equation implies that $\tau=\mathbf{s}^{*} u$ for some nonvanishing function $u$ on $\Gamma_{0}$, and the second that $\phi=\frac{\mathbf{s}^{*} u}{\mathbf{t}^{*} u}$, as claimed.

Remark 1.6. The change in ii) corresponds to a $u^{-1}$-conformal change on the base $\Gamma_{0}$ and a $\left(\mathbf{s}^{*} u\right)^{-1}$-conformal change on $\Gamma$.

It is not hard to verify that a contact groupoid action is also a conformal contact groupoidaction. Now we prove the converse:

Lemma 1.7. Let $\Phi: M_{J} \times_{\mathbf{t}} \Gamma \rightarrow M$ be a conformal contact groupoidaction. Then

i) Given a triple $\left(\Gamma, f, \theta_{\Gamma}\right)$ as in Lemma 1.5 , there is a unique contact 1-form $\theta_{M}$ on $M$ such that $\Phi$ is a contact groupoid action;

ii) $\left(\Gamma, \hat{f}, \hat{\theta}_{\Gamma}\right)$ and $\left(M, \hat{\theta}_{M}\right)$ are another such pair if and only if $\hat{f}=f \frac{\mathbf{s}^{*} u}{t^{*} u}, \hat{\theta}_{\Gamma}=$ $\mathbf{s}^{*} u \cdot \theta_{\Gamma}$ and $\hat{\theta}_{M}=J^{*} u \cdot \theta_{M}$.

Proof. Given a triple $\left(\Gamma, f, \theta_{\Gamma}\right)$ as in i), let $E_{\Gamma}$ be the Reeb vector field of $\Gamma$ corresponding to the 1 -form $\theta_{\Gamma}$. Define a vector field on $M$ by

$$
E_{M}(m):=0\left(m g^{-1}\right) \cdot E_{\Gamma}(g) .
$$

This vector field is well defined since using the $f$-multiplicativity of $\theta_{\Gamma}$ one can show that $E_{\Gamma}\left(g^{\prime}\right)=0\left(g^{\prime} g^{-1}\right) \cdot E_{\Gamma}(g)$ whenever $\mathbf{s}(g)=\mathbf{s}\left(g^{\prime}\right)$. By equation (25) there exists a (unique) contact 1-form $\theta_{M}$ with kernel $\mathcal{H}_{M}$ and $E_{M}$ as Reeb vector field. 
Endowing $M \times \mathbb{R} \times \Gamma \times \mathbb{R} \times M$ with the contact structure as in Lemma 3.3 we obtain as contact hyperplane

$$
\begin{aligned}
\mathcal{H}= & \left(\mathcal{H}_{M} \times 0 \times \mathcal{H}_{M} \times 0 \times \mathcal{H}_{M}\right) \oplus \operatorname{span}\left\{\frac{\partial}{\partial a}\right\} \oplus \operatorname{span}\left\{\frac{\partial}{\partial b}\right\} \\
& \oplus \operatorname{span}\left\{\left(E_{M}, 0,0,0, f e^{-a} E_{M}\right)\right\} \oplus \operatorname{span}\left\{\left(0,0, E_{\Gamma}, 0, e^{-b} E_{M}\right)\right\} .
\end{aligned}
$$

Denote the graph of the action $\Phi$ by $\mathcal{A}$. By i) in Definition 1.2 ,

$$
\operatorname{dim}\left(\left(\mathcal{H}_{M} \times 0 \times \mathcal{H}_{\Gamma} \times 0 \times \mathcal{H}_{M}\right) \cap T \mathcal{A}\right) \geq k+n-1,
$$

where $\operatorname{dim} M=k$ and $\operatorname{dim} \Gamma=2 n+1$. Again using the $f$-multiplicativity of $\theta_{\Gamma}$ (equation (3)) and the fact that $\mathbf{t}$ is $-f$-Jacobi, one can show that

$$
E_{\Gamma}(h) \cdot\left(X_{-f}\right)_{\mathcal{H}_{\Gamma}}(g)=f(g) E_{\Gamma}(h g)
$$

whenever $\mathbf{s}(h)=\mathbf{t}(g)$, where $\left(X_{-f}\right)_{\mathcal{H}_{\Gamma}}$ is the projection of $X_{-f}$ onto $\mathcal{H}_{\Gamma}$. This together with the definition of $E_{M}$ imply that

$$
\left(E_{M}, 0,\left(X_{-f}\right)_{\mathcal{H}_{\Gamma}}, 0, f E_{M}\right) \text { and }\left(0,0, E_{\Gamma}, 0, E_{M}\right) \in \mathcal{H} \cap T \mathcal{A} \text {. }
$$

Therefore with these two more vectors, we have $\operatorname{dim}(\mathcal{H} \cap T \mathcal{A}) \geq k+n+1$. On the other hand $T \mathcal{A}$ has dimension $k+n+1$, so we have $T \mathcal{A} \subset \mathcal{H}$ and $\mathcal{A}$ is a Legendrian submanifold. By Lemma 3.3, the action is a contact groupoid action. The uniqueness follows because by equation (6) for any contact groupoid action we have $0 \cdot E_{\Gamma}=E_{M}$.

To prove ii) notice that the expressions for $\hat{f}$ and $\hat{\theta}_{\Gamma}$ were derived in Lemma 1.5. By the proof of i) the expression for $\hat{\theta}_{M}$ is determined by its Reeb vector field $\hat{E}_{M}:=0 \cdot \hat{E}_{\Gamma}=0 \cdot \frac{1}{\mathbf{s}^{*} u} E_{\Gamma}=\frac{1}{J^{*} u} E_{M}$, where $\hat{E}_{\Gamma}$ denotes the Reeb vector field of $\hat{\theta}_{\Gamma}$.

Now the proof of Theorem 1.4 is straightforward.

Proof of Theorem 1.4. Let $\left(\Gamma, \mathcal{H}_{\Gamma}\right)$ be a contact-structure groupoid. Lemma 1.5 tells us what the "compatible" choices of pairs $\left(\theta_{\Gamma}, f\right)$ are on $\Gamma$. Now let $\left(M, \mathcal{H}_{M}\right)$ be a manifold with a contact structure and $\Phi$ be a conformal contact groupoidaction of $\left(\Gamma, \mathcal{H}_{\Gamma}\right)$ on $\left(M, \mathcal{H}_{M}\right)$. Lemma 1.7 tells us that for each pair $\left(\theta_{\Gamma}, f\right)$ there is a unique choice for $\theta_{M}$ that makes $\Phi$ a contact groupoid action. If we make a choice of pair $\left(\theta_{\Gamma}, f\right)$ and consider the corresponding form $\theta_{M}$, we obtain by Theorem 4.4 a Jacobi structure on $M / \Gamma$ by requiring that $p r: M \rightarrow M / \Gamma$ be a $-F$-conformal Jacobi map, where $F$ is some $f$-multiplicative function on $M$.

Let $\left(\hat{\theta}_{\Gamma}:=\mathbf{s}^{*} u \cdot \theta_{\Gamma}, \hat{f}:=f \frac{\mathbf{s}^{*} u}{\mathbf{t}^{*} u}, \hat{\theta}_{M}:=J^{*} u \cdot \theta_{M}\right)$ be another set of data as above. It is straightforward to check that $\hat{F}:=J^{*} u \cdot F$ is a $\hat{f}$-multiplicative function. The corresponding Jacobi structure on $M / \Gamma$ is obtained by requiring that $p r$ be a $-\hat{F}$ conformal Jacobi map with respect to the contact form $\hat{\theta}_{M}=J^{*} u \cdot \theta_{M}$, i.e. that it be a Jacobi map with respect to the Jacobi structure on $M$ obtained from the original on 17 twisting by $-\hat{F} \cdot\left(J^{*} u\right)^{-1}=-F$. Therefore the two Jacobi structures on $M / \Gamma$ obtained above are identical. This shows that the conformal class is independent of all the choices we made.

\footnotetext{
${ }^{17}$ That is, the one corresponding to $\theta_{M}$.
} 


\section{APPENDIX II-ON LEFT/RIGHT ACTIONS AND SIGN CONVENTIONS}

The definition of contact groupoids we adopted (Definition 2.1) allows one to define only right actions (Definition 3.1). In this appendix we describe how to switch from such a groupoid to one for which we can naturally define left actions.

We start by describing a setting that includes both kinds of groupoids Daz97. Given a conformal contact groupoid $\left(\Gamma, \mathcal{H}_{\Gamma}\right)$ for which the contact structure is co-orientable (see Definition 1.1 in Appendix I), one can choose a corresponding contact form $\theta$ and two multiplicative functions $f_{L}, f_{R}: \Gamma \rightarrow \mathbb{R}-\{0\}$ such that the multiplication $\omega$ satisfie 18

$$
\omega^{*}(\theta)=p r_{2}^{*}\left(f_{R}\right) p r_{1}^{*}(\theta)+p r_{1}^{*}\left(f_{L}\right) p r_{2}^{*} h(\theta) .
$$

Furthermore $\Gamma_{0}$ can be given a Jacobi structure so that $\mathbf{s}$ is a $f_{L}$-Jacobi map and $\mathbf{t}$ an $-f_{R^{-}}$Jacobi map 19 Clearly imposing that $\mathbf{s}$ be $-f_{L^{-}}$Jacobi and $\mathbf{t}$ be $f_{R^{-}}$-Jacobi endows $\Gamma_{0}$ with a Jacobi structure which is the negative of the above.

One can always arrang 20 that either $f_{L} \equiv 1$ or $f_{R} \equiv 1$. We will adopt the following conventions for the induced Jacobi structure on $\Gamma_{0}$ :

a) If $f_{L} \equiv 1$ ("right contact groupoid"), then $\mathbf{s}$ is a Jacobi map.

b) If $f_{R} \equiv 1$ ("left contact groupoid"), then $\mathbf{t}$ is a Jacobi map.

Notice that convention a) above is the one used by Kebrat and Souici in KSB93. and the one we followed in this paper (see Definition 2.1).

Now recall that if $\Gamma \rightrightarrows \Gamma_{0}$ is any Lie groupoid and $\Phi_{r}: M_{J} \times_{\mathbf{t}} \Gamma \rightarrow M$ is a right groupoid action on $J: M \rightarrow \Gamma_{0}$, then by $\Phi_{l}(g, m)=\Phi_{r}\left(m, g^{-1}\right)$ we obtain a left groupoid action $\Phi_{l}: \Gamma_{\mathbf{s}} \times_{J} M \rightarrow M$ on $J$. Suppose we are given a "right contact groupoid", i.e. a tuple $\left(\Gamma, \theta_{r}, 1, f_{r}\right)$ satisfying (26), and suppose $\Phi_{r}$ as above is a contact groupoid action on some contact manifold $\left(M, \theta_{M}\right)$. Then $\Phi_{l}$ satisfies

$$
\Phi_{l}^{*}\left(\theta_{l}\right)=p r_{\Gamma}^{*}\left(\theta_{l}\right)+p r_{\Gamma}^{*}\left(f_{l}\right) p r_{M}^{*}\left(\theta_{M}\right),
$$

where $\theta_{l}:=i^{*} \theta_{r}=-\frac{1}{f_{r}} \theta_{r}$ and $f_{l}:=i^{*} f_{r}=\frac{1}{f_{r}}$. The new structure $\left(\Gamma, \theta_{l}, f_{l}, 1\right)$ satisfies (26), so we can define it to be the "left contact groupoid" associated to $\left(\Gamma, \theta_{r}, 1, f_{r}\right)$. Furthermore we take (26) to be the defining equation for left contact groupoid actions.

Notice that switching from a "right" to "left" contact groupoid does not change the underlying conformal contact groupoid $\left(\Gamma, \mathcal{H}_{\Gamma}\right)$. Furthermore, assuming our conventions a) and b) above, it does not change the Jacobi structure induced on $\Gamma_{0}:$ indeed $\mathbf{s}:\left(\Gamma, \theta_{r}=-\frac{1}{f_{l}} \theta_{l}\right) \rightarrow \Gamma_{0}$ is a Jacobi map exactly when $\mathbf{s}:\left(\Gamma, \theta_{l}\right) \rightarrow \Gamma_{0}$ is a $-f_{l}$-Jacobi map, which happens exactly when $\mathbf{t}:\left(\Gamma, \theta_{l}\right) \rightarrow \Gamma_{0}$ is a Jacobi map.

We conclude this appendix by describing how our conventions a) and b) fit with choices of Lie algebroids for $\Gamma$. Recall that a Lie algebroid is a vector bundle $E \rightarrow N$ together with a bundle map (the anchor) $E \rightarrow T N$ and a Lie bracket on its space of sections satisfying certain conditions (see [CdSW99]). Given any Lie groupoid $\Gamma \rightrightarrows \Gamma_{0}$, there are two associated Lie algebroids: one is ker $\left.\mathbf{t}_{*}\right|_{\Gamma_{0}}$, with Lie bracket induced by the bracket of left-invariant vector fields on $\Gamma$ and with anchor $\mathbf{s}_{*}$. The other one is $\left.\operatorname{ker} \mathbf{s}_{*}\right|_{\Gamma_{0}}$ with anchor $\mathbf{t}_{*}$. Under the identification $\left.\left.\operatorname{ker} \mathbf{t}_{*}\right|_{\Gamma_{0}} \cong T \Gamma\right|_{\Gamma_{0}} /\left.T \Gamma_{0} \cong \operatorname{ker} \mathbf{s}_{*}\right|_{\Gamma_{0}}$ (which is given by $-i_{*}$ for $i: \Gamma \rightarrow \Gamma$ the

\footnotetext{
${ }^{18}$ See Proposition 4.1 in Daz97.

${ }^{19}$ See Theorem 4.1ii in Daz97.

${ }^{20}$ See the proof of Proposition 4.1 of Daz97.
} 
inversion), the two algebroid structures are anti-isomorphic 21 Notice that this implies that $i_{*}:\left.\left.\operatorname{ker} \mathbf{t}_{*}\right|_{\Gamma_{0}} \rightarrow \operatorname{ker} \mathbf{s}_{*}\right|_{\Gamma_{0}}$ is a Lie algebroid isomorphism, but we will not use this fact.

A right action of $\Gamma$ on a manifold $M$ with moment map $J: M \rightarrow \Gamma_{0}$ clearly induces by differentiation an algebroid action of ker $\left.\mathbf{t}_{*}\right|_{\Gamma_{0}}$, whereas a left groupoid action induces an action of ker $\left.\mathbf{s}_{*}\right|_{\Gamma_{0}}$. In this sense ker $\left.\mathbf{t}_{*}\right|_{\Gamma_{0}}$ is the preferred algebroid for "right contact groupoids", and ker $\left.\mathbf{s}_{*}\right|_{\Gamma_{0}}$ for "left contact groupoids".

Now let $\left(\Gamma, \theta, f_{L}, f_{R}\right)$ be a groupoid satisfying (26). There are two natural vector bundle isomorphism: 22 from the Lie algebroid $T^{*} \Gamma_{0} \times \mathbb{R}$ of the the Jacobi manifold $\Gamma_{0}$ to the two algebroids of $\Gamma$ :

$$
T^{*} \Gamma_{0} \times\left.\mathbb{R} \rightarrow \operatorname{ker} \mathbf{t}_{*}\right|_{\Gamma_{0}},\left(\varphi_{1}, \varphi_{0}\right) \mapsto \mathbf{s}^{*} \varphi_{0} \cdot X_{f_{L}}+f_{L} \cdot \sharp \Lambda \mathbf{s}^{*} \varphi_{1}
$$

and

$$
T^{*} \Gamma_{0} \times\left.\mathbb{R} \rightarrow \operatorname{ker} \mathbf{s}_{*}\right|_{\Gamma_{0}},\left(\varphi_{1}, \varphi_{0}\right) \mapsto \mathbf{t}^{*} \varphi_{0} \cdot X_{f_{R}}+f_{R} \cdot \sharp \Lambda \mathbf{t}^{*} \varphi_{1},
$$

and it is a straightforward computation using (26) to show that $-i_{*}:\left.\operatorname{ker} \mathbf{t}_{*}\right|_{\Gamma_{0}} \rightarrow$ $\left.\operatorname{ker} \mathbf{s}_{*}\right|_{\Gamma_{0}}$ intertwines them.

If we endow $\Gamma_{0}$ with a Jacobi structure so that $\mathbf{s}$ is a $f_{L^{-}}$Jacobi map and $\mathbf{t}$ a $-f_{R^{-}}$ Jacobi map, then the map (28) is an isomorphims of Lie algebroids 23 Therefore when $\Gamma$ is a "right contact groupoid" following convention a) we obtain a natural isomorphism between the algebroid of $\Gamma_{0}$ and the preferred algebroid of $\Gamma$. The analogous statement for "left contact groupoids" holds as well.

\section{ACKNOWLEDGEMENTS}

We thank our advisor A. Weinstein, as well as M. Crainic, Y. Eliashberg, M. Harada, T. Holm, A. Knutson, E. Lebow, E. Lerman and C. Willett for helpful discussions.

\section{REFERENCES}

[Alb89] Claude Albert, Le théorème de réduction de Marsden-Weinstein en géométrie cosymplectique et de contact, J. Geom. Phys. 6 (1989), no. 4, 627-649. MR91k:58033

[BCWZar] Henriques Bursztyn, Marius Crainic, Alan Weinstein, and Chenchang Zhu, Integration of twisted Dirac brackets, Duke. Math. J. 123 (2004), no. 3, 549-607. MR2068969

[Bla02] David E. Blair, Riemannian geometry of contact and symplectic manifolds, Progress in Mathematics, vol. 203, Birkhäuser Boston, Inc., Boston, MA, 2002. MR2002m:53120

[BtD95] Theodor Bröcker and Tammo tom Dieck, Representations of compact Lie groups, Graduate Texts in Mathematics, vol. 98, Springer-Verlag, New York, 1995, Translated from the German manuscript, Corrected reprint of the 1985 translation. MR 97i:22005

[CdS01] Ana Cannas da Silva, Lectures on symplectic geometry, Lecture Notes in Mathematics, vol. 1764, Springer-Verlag, Berlin, 2001. MR2002i:53105

[CdSW99] Ana Cannas da Silva and Alan Weinstein, Geometric models for noncommutative algebras, Berkeley Mathematics Lecture Notes, vol. 10, American Mathematical Society, Providence, RI, 1999. MR2001m:58013

[CDW87] A. Coste, P. Dazord, and A. Weinstein, Groupoïdes symplectiques, Publications du Département de Mathématiques. Nouvelle Série. A, Vol. 2, Publ. Dép. Math. Nouvelle Sér. A, vol. 87, Univ. Claude-Bernard, Lyon, 1987, pp. i-ii, 1-62. MR90g:58033

[CZ] Marius Crainic and Chenchang Zhu, Integrability of Jacobi structures, arXiv:math.DG/0403268.

\footnotetext{
${ }^{21}$ See Theorem 9.15 in Vai94.

${ }^{22}$ See Proposition 4.3 and the remarks on page 443 and page 446 in [Daz97.

${ }^{23}$ See the second part of Theorem 4.1 of [Daz97].
} 
[Daz97] Pierre Dazord, Sur l'intégration des algèbres de Lie locales et la préquantification, Bull. Sci. Math. 121 (1997), no. 6, 423-462. MR99g:58057

[dLLMP03] Manuel de León, Belén López, Juan C. Marrero, and Edith Padrón, On the computation of the Lichnerowicz-Jacobi cohomology, J. Geom. Phys. 44 (2003), no. 4, 507-522. MR2003m:53145

[GGK02] Victor Guillemin, Viktor Ginzburg, and Yael Karshon, Moment maps, cobordisms, and Hamiltonian group actions, Mathematical Surveys and Monographs, vol. 98, American Mathematical Society, Providence, RI, 2002, Appendix J by Maxim Braverman. MR 2003m:53149

[Kir76] A. Kirillov, Local Lie algebras, Russian Math. Surveys 31 (1976), 55-75. MR0438390 (55:11304a)

[Kos70] Bertram Kostant, Quantization and unitary representations. I. Prequantization, Lectures in modern analysis and applications, III, Springer, Berlin, 1970, pp. 87-208. Lecture Notes in Math., Vol. 170. MR 45:3638

[KSB93] Yvan Kerbrat and Zoubida Souici-Benhammadi, Variétés de Jacobi et groupoïdes de contact, C. R. Acad. Sci. Paris Sér. I Math. 317 (1993), no. 1, 81-86. MR94g:58072

[Lic78] André Lichnerowicz, Les variétés de Jacobi et leurs algèbres de Lie associées, J. Math. Pures Appl. (9) 57 (1978), no. 4, 453-488. MR80m:58016

[MM03] I. Moerdijk and J. Mrčun, Introduction to foliations and Lie groupoids, Cambridge Studies in Advanced Mathematics, vol. 91, Cambridge University Press, Cambridge, 2003. MR2012261

[MW74] Jerrold Marsden and Alan Weinstein, Reduction of symplectic manifolds with symmetry, Rep. Mathematical Phys. 5 (1974), no. 1, 121-130. MR.53:6633

[MW88] Kentaro Mikami and Alan Weinstein, Moments and reduction for symplectic groupoids, Publ. Res. Inst. Math. Sci. 24 (1988), no. 1, 121-140. MR.90c:58060

[SW99] Richard Schoen and Jon Wolfson, Minimizing volume among Lagrangian submanifolds, Proceedings of Symposia in Pure Mathematics, vol. 65, American Mathematical Society, Providence, RI, 1999. MR.1662755 (99k:53130)

[Vai94] Izu Vaisman, Lectures on the geometry of Poisson manifolds, Progress in Mathematics, vol. 118, Birkhäuser Verlag, Basel, 1994. MR95h:58057

[Wil02] Christopher Willett, Contact reduction, Trans. Amer. Math. Soc. 354 (2002), no. 10, 4245-4260 (electronic). MR 2003m:53152

[Xu91] Ping Xu, Morita equivalence of Poisson manifolds, Comm. Math. Phys. 142 (1991), no. 3, 493-509. MR.93a:58069

Department of Mathematics, University of California, Berkeley, California 94720

Current address: Institut für Mathematik, Universität Zürich-Irchel, 8057 Zürich, Switzerland

E-mail address: zambon@math.unizh.ch

Department of Mathematics, University of California, Berkeley, California 94720

Current address: D-MATH, ETH-Zentrum, CH-8092 Zürich, Switzerland

E-mail address: zhu@math.ethz.ch 